OPEN ACCESS

Edited by:

Laigeng Li,

Institute of Plant Physiology

and Ecology, China

Reviewed by:

Chengbin Xiang,

University of Science and Technology

of China, China

Ahmad H. Kabir,

University of Rajshahi, Bangladesh

*Correspondence:

Fengming Song

fmsong@zju.edu.cn

Specialty section: This article was submitted to

Plant Physiology,

a section of the journa

Frontiers in Plant Science

Received: 20 August 2015

Accepted: 06 January 2016

Published: 22 January 2016

Citation:

Hong Y, Zhang H, Huang L, Li D and Song $F$ (2016) Overexpression

of a Stress-Responsive NAC Transcription Factor Gene ONAC022 Improves Drought and Salt Tolerance in Rice. Front. Plant Sci. 7:4. doi: 10.3389/fpls.2016.00004

\section{Overexpression of a} Stress-Responsive NAC Transcription Factor Gene ONAC022 Improves Drought and Salt Tolerance in Rice

\author{
Yongbo Hong, Huijuan Zhang, Lei Huang, Dayong Li and Fengming Song* \\ National Key Laboratory for Rice Biology, Institute of Biotechnology, Zhejiang University, Hangzhou, China
}

The NAC transcription factors play critical roles in regulating stress responses in plants. However, the functions for many of the NAC family members in rice are yet to be identified. In the present study, a novel stress-responsive rice NAC gene, ONAC022, was identified. Expression of ONAC022 was induced by drought, high salinity, and abscisic acid (ABA). The ONAC022 protein was found to bind specifically to a canonical NAC recognition cis-element sequence and showed transactivation activity at its C-terminus in yeast. The ONAC022 protein was localized to nucleus when transiently expressed in Nicotiana benthamiana. Three independent transgenic rice lines with overexpression of ONAC022 were generated and used to explore the function of ONAC022 in drought and salt stress tolerance. Under drought stress condition in greenhouse, soil-grown ONAC022-overexpressing (N22oe) transgenic rice plants showed an increased drought tolerance, leading to higher survival ratios and better growth than wild-type (WT) plants. When grown hydroponically in Hogland solution supplemented with $150 \mathrm{mM} \mathrm{NaCl}$, the $\mathrm{N} 22 \mathrm{oe}$ plants displayed an enhanced salt tolerance and accumulated less $\mathrm{Na}^{+}$in roots and shoots as compared to WT plants. Under drought stress condition, the N22oe plants exhibited decreased rates of water loss and transpiration, reduced percentage of open stomata and increased contents of proline and soluble sugars. However, the N22oe lines showed increased sensitivity to exogenous ABA at seed germination and seedling growth stages but contained higher level of endogenous ABA. Expression of some ABA biosynthetic genes (OsNCEDs and OsPSY), signaling and regulatory genes (OsPP2C02, OsPP2C49, OsPP2C68, OsbZIP23, OsAP37, OsDREB2a, and OsMYB2), and late stress-responsive genes (OsRAB21, OsLEA3, and OsP5CS1) was upregulated in N22oe plants. Our data demonstrate that ONAC022 functions as a stress-responsive NAC with transcriptional activator activity and plays a positive role in drought and salt stress tolerance through modulating an ABA-mediated pathway.

Keywords: abscisic acid (ABA), drought tolerance, NAC transcription factor, ONAO22, salt tolerance, rice (Oryza sativa L.) 


\section{INTRODUCTION}

Plants have developed an array of sophisticated mechanisms at multiple levels to cope with unfavorable environmental stresses, such as drought and high salinity (Yamaguchi-Shinozaki and Shinozaki, 2006). Upon perception of external stress signals, a complicated signaling network is effectively and timely initiated, which ultimately reprograms the expression of a large set of stress-responsive genes (Zhu, 2002; Shinozaki and YamaguchiShinozaki, 2007; Golldack et al., 2014; Nakashima et al., 2014). This expression reprograming of stress-responsive genes at genome-wide level often activates various cellular, physiological, biochemical, and metabolic processes, including stomatal closure, repression of cell growth and photosynthesis, activation of respiration, and accumulation of compatible osmolytes, such as proline and soluble sugars, which protect plants from damages and thus increase the chance of survival (Shinozaki and Yamaguchi-Shinozaki, 2007; Hu and Xiong, 2014).

Recent extensive genetic and molecular studies using knockout/knockdown mutants and overexpression transgenic lines in model plants as well as in crop plants have demonstrated that many transcription factors (TFs) belonging to the NAC, AP2/ERF, MYB, WRKY, bZIP, homeodomain, bHLH, NF-Y, and CAMTA families play important roles in plant responses to abiotic and biotic stresses (Ariel et al., 2007; Chen et al., 2012; Mizoi et al., 2012; Puranik et al., 2012; Rushton et al., 2012; Licausi et al., 2013; Nuruzzaman et al., 2013; Castilhos et al., 2014; Nakashima et al., 2014; Shao et al., 2015). The NAC (NAM, AFAT, and $\underline{\text { CUC) }}$ proteins are characterized with a conserved region, called NAC domain, at their N-terminals and a highly divergent C-terminus (Olsen et al., 2005). Genome-based bioinformatics analyses have showed that the NAC proteins constitute a large plant-specific family with more than 100 members (Ooka et al., 2003; Fang et al., 2008; Nuruzzaman et al., 2010; Le et al., 2011). A large number of the NAC TFs have been characterized for their roles in plant abiotic stress responses (Puranik et al., 2012; Nuruzzaman et al., 2013). Transcriptional profiling analysis revealed that at least 33 Arabidopsis NAC genes were responsible to abiotic stresses including high salinity (Jiang and Deyholos, 2006). Similarly, it was also found that a large portion of the rice NAC family exhibited overlapping expression patterns in rice under various abiotic and biotic stresses (Nuruzzaman et al., 2015; Sun et al., 2015). Six rice NAC genes have been shown to be involved in defense response against pathogen infection, e.g., ONAC048 (OsNAC6), ONAC048 (OsNAC111), ONAC122, and ONAC131 in regulating defense response against Magnaporthe oryzae causing blast disease (Nakashima et al., 2007; Sun et al., 2013; Yokotani et al., 2014), ONAC054 (RIM1) with negative effect on resistance to rice dwarf virus (Yoshii et al., 2009) and ONAC068 (OsNAC4) as a positive regulator of hypersensitive cell death (Kaneda et al., 2009). The involvement of NAC TFs in rice abiotic stress response was extensively explored and seven NAC genes have been characterized to play important roles in abiotic stress tolerance in rice. For example, overexpression of ONAC002 (SANC1/OsNAC9), ONAC048 (SNAC2/OsNAC6), ONAC009 (OsNAC5), ONAC122 (OsNAC10), ONAC045, or
ONAC058 (OsNAP) improved significantly the drought and salinity tolerance in transgenic rice (Hu et al., 2006, 2008; Nakashima et al., 2007; Zheng et al., 2009; Jeong et al., 2010, 2013; Takasaki et al., 2010; Song et al., 2011; Redillas et al., 2012; Chen et al., 2014; Liang et al., 2014) and some of these transgenic rice lines showed increased drought tolerance under severe drought stress conditions without any adverse effect on yield or even with yield increase (Hu et al., 2006; Jeong et al., 2010, 2013; Redillas et al., 2012; Chen et al., 2014). Abscisic acid (ABA) is a well-known stress hormone that coordinates the complex networks of stress responses. ABA-mediated signaling pathway plays important roles in abiotic stress responses regulated by several NAC TFs in transgenic rice plants, as demonstrated by the hypersensitivity to ABA (Hu et al., 2006, 2008; Chen et al., 2014), upregulated expression of ABA biosynthesis-related genes (Jeong et al., 2010; Redillas et al., 2012), increased endogenous ABA level (Liang et al., 2014), and upregulated expression of a set of ABA-responsive stress-related genes (Nakashima et al., 2007; Hu et al., 2008; Jeong et al., 2010, 2013; Takasaki et al., 2010; Redillas et al., 2012; Chen et al., 2014). In addition, altered stomatal movement or root system regulated by overexpression of ONACO02 (SANC1/OsNAC9), ONAC009 (OsNAC5), or ONAC122 (OsNAC10) was also found to be involved in the improved abiotic stress tolerance of the transgenic plants $(\mathrm{Hu}$ et al., 2006; Jeong et al., 2010, 2013; Takasaki et al., 2010; Redillas et al., 2012).

We are interested to characterize novel $\mathrm{TF}$ genes that regulate plant stress response and try to generate stress-resistant/tolerant crops through transgenic approach. In our previous study, we characterized a number of stress-responsive ONAC genes in rice response to biotic and abiotic stresses using publicly available microarray data (Sun et al., 2013, 2015) and noticed that one ONAC gene, ONAC022 (LOC_Os03g04070), was significantly induced by several abiotic and biotic stress treatments. In the present study, we performed a detailed functional analysis by overexpression of ONAC022 in transgenic rice to explore its roles in abiotic stress tolerance.

\section{MATERIALS AND METHODS}

\section{Plant Growth and Treatments}

Rice (Oryza sativa L.) cultivar Yuanfengzao was used for analyses of gene expression in response to abiotic stress and ABA treatment whereas cultivar Xiushui 134 for generation of transgenic lines and phenotype analyses. Seedlings were grown in a soil mix (clay is mixed with soil at 3:1 ratio) in a growth room under daily cycle of $28^{\circ} \mathrm{C} 14 \mathrm{~h}$ light (>3000 lux) $/ 22^{\circ} \mathrm{C}$ $10 \mathrm{~h}$ dark or in a greenhouse with natural sunlight. For hormone treatment, 2-week-old seedlings grown in a growth room were treated by spraying with $100 \mu \mathrm{M} \mathrm{ABA}$ or with equal volume of solution containing only $0.1 \%$ ethanol and $0.02 \%$ Tween-20 as controls. For drought stress treatment, seedlings were put on lab blenches without water supply or on water-saturated filter papers in Petri dishes as controls. For salt stress treatment, seedlings were irrigated with $200 \mathrm{mM} \mathrm{NaCl}$ solution. Leaf samples were collected and stored at $-80^{\circ} \mathrm{C}$ until use. 


\section{Cloning and Sequence Analysis of ONACO22}

Based on the predicted sequence of ONAC022 (LOC_Os03g04070) in Rice Genome Annotation database and a full-length cDNA (AK107090) in GenBank database, the coding sequence of ONACO22 was amplified using a pair of gene-specific primers (Supplementary Table S1) and cloned into pMD19-T vector, yielding plasmid pMD19-ONAC022. After confirmation by sequencing, plasmid pMD19-ONAC022 was used as a template for all experiments. Multiple sequence alignment was performed using ClustalW program in the Lasergene software. Phylogenetic tree analysis with other rice NAC proteins retrieved from the Rice Genome Annotation database was performed using neighbor-joining method in MEGA5 software with 1000 replications. The promoter sequence of the ONAC022 gene (1500 bp upstream from the transcription start site) was retrieved and searched for putative cis-elements at the PLACE database (http://www.dna.affrc.go.jp/PLACE/signalscan.html).

\section{Subcellular Localization Assay}

The coding sequence of ONAC022 was amplified using a pair of gene-specific primers (Supplementary Table S1) and inserted into vector pFGC-EGFP at $\mathrm{BamHI} / \mathrm{XbaI}$ sites, yielding plasmid pFGC-GFP-ONAC022. This plasmid and the pFGC-EGFP empty vector were transformed into Agrobacterium tumefaciens GV3101 and the agrobacteria were infiltrated separately into leaves of Nicotiana benthamiana plants expressing a red nuclear marker RFP-H2B protein (Chakrabarty et al., 2007) using 1-mL needless syringes. After agroinfiltration, the plants were grown in a growth room under $25^{\circ} \mathrm{C}$ for $48 \mathrm{~h}$. GFP fluorescence signals were excited at $488 \mathrm{~nm}$ and detected under a Zeiss LSM 510 Meta confocal laser scanning microscope (Oberkochen, Germany) using a $500-530 \mathrm{~nm}$ emission filter.

\section{DNA Binding and Transactivation Activity Assays}

For DNA binding activity assay, the coding sequence of ONAC022 was amplified using a pair of gene-specific primers (Supplementary Table S1) and inserted into pGEX-6p-1 (GE Healthcare, Piscataway, NJ, USA) at BamHI/EcoRI sites, yielding plasmid pGEX-6p1-ONAC022, which was then transformed into Escherichia coli strain BL21. GST-fused ONAC022 recombinant protein was purified using glutathione resin column (Genscript, Shanghai, China) according to the manufacturer's protocol. A 28 bp cis-element fragment wNACRS (ATCGCATGTGGAGCACGGAGCACGTTTT, the core sequences underlined; Xie et al., 2000; Trans et al., 2004) and a mutated fragment mNACRS (ATCGAAAAAAGAGAAAAGAGAAAA TTTT, the mutated nucleotides underlined) were labeled with biotin. Electrophoretic mobility shift assay (EMSA) was performed using a chemiluminescent EMSA Kit (Beyotime Biotechnology, Haimen, China). Binding reactions were conducted in a total of $10 \mu \mathrm{L}$ volume containing $5 \mathrm{x}$ EMSA buffer, $2 \mu \mathrm{g}$ recombinant ONAC022 protein or GST protein (as a negative control) and $1 \mu \mathrm{L}$ biotin-labeled wNACRS or mNACRS probe. For the competitive reactions, excess unlabeled
wNACRS or mNACRS probe (in excess of 200 times) was added and incubated for $20 \mathrm{~min}$ before addition of the labeled wNACRS probe. The reaction mixtures were separated on $8 \%$ native PAGE and transferred onto nitrocellulose membranes. Signals from the probes were detected according to the manufacturer's protocol. For transactivation activity assay, the coding sequence of ONAC022 was amplified using a pair of gene-specific primers (Supplementary Table S1) and fused in-frame to yeast GAL4 DNA binding domain in vector pBD-GAL4Cam, yielding plasmid pBD-ONAC022. Plasmid pBD-ONAC022 and $\mathrm{pBD}$ empty vector were transformed into yeast strain AH109. The transformed yeasts were plated on $\mathrm{SD} / \mathrm{Trp}^{-}$or $\mathrm{SD} / \mathrm{Trp}^{-}$ $\mathrm{His}^{-}$medium and incubated for 3 days at $30^{\circ} \mathrm{C}$, followed by addition of $\mathrm{x}-\alpha$-gal. Transactivation activity of the fused proteins was evaluated according to the growth and production of blue pigments after addition of $\mathrm{x}$ - $\alpha$-gal on $\mathrm{SD} / \mathrm{Trp}^{-} \mathrm{His}^{-}$ medium.

\section{Generation and Characterization of N22oe Lines}

The coding sequence of ONACO22 was amplified using ONAC022-F-OE and ONAC022-R-OE (Supplementary Table S1) and inserted into binary vector pCoUm (Zhang et al., 2008) under the control of a maize ubiquitin promoter, yielding plasmid pCoUm-Ubi::ONAC022. The resulting construct was introduced into rice calli of cultivar Xiushuil34 through Agrobacteriummediated transformation. T2 generation of the obtained N22oe lines was screened by planting seeds on 1/2 MS medium supplemented with $50 \mu \mathrm{g} / \mathrm{L}$ hygromycin (Hgr) and lines showing 3:1 (Hgr-resistant:Hgr-susceptible) segregation were selected as putative transgenic lines with single-copy of the transgene. Seeds from individual lines of T3 generation were observed on selective medium and those that showed $100 \%$ resistance to Hgr were selected as homozygous lines. Homozygous lines with singlecopy of transgene were used for all experiments. To further characterize these single-copy transgenic lines, genomic DNA was extracted with two volumes of $2 \mathrm{x}$ CTAB extraction buffer $(2 \%$ CTAB, 10 mM Tris- $\mathrm{HCl}$ pH8.0, 20 mM EDTA pH8.0, $1.4 \mathrm{M} \mathrm{NaCl}$, $2 \%$ 2-ME). Fifty micrograms of genomic DNA were digested completely with EcoRI, separated by electrophoresis on a $0.8 \%$ agarose gel, and transferred by capillary action overnight onto a Hybond- $\mathrm{N}^{+}$nylon membrane (Amersham Biosciences, Little Chalfont, UK) using 20X SSC solution. A $589 \mathrm{bp}$ fragment of the HptII gene was amplified using a pair of primers HptII-Probe-F and HptII-Probe-R (Supplementary Table S1) and labeled with DIG by the random priming method using a DIG High Prime DNA Labeling and Detection kit (Roche Diagnostics, Shanghai, China). Prehybridization, hybridization, and detection were performed according to the manufacturer's recommendations.

\section{Phenotype Analyses for Drought and Salt Tolerance and ABA sensitivity}

For drought tolerance assay, ten 4-week-old N22oe and wild-type (WT) seedlings were grown in the same pot with three replicates and were subjected to drought stress treatment by withholding watering for 15 days, followed by recovery with normal water 
supply for another 7 days. The survival ratio and fresh weight of the plants were calculated at 12 days after re-watering. Plants that were green and healthy young leaves after re-watering were regarded as survivals. Survival ratio was calculated as the ratio of number of survived plants over the total number of treated plants. For salt tolerance assay, 100 seeds were germinated on 1/2 MS medium supplemented with or without $150 \mathrm{mM} \mathrm{NaCl}$ under $28^{\circ} \mathrm{C} / 25^{\circ} \mathrm{C}$ (day/night) with a $12 \mathrm{~h}$ photoperiod. At 6 days after germination, shoot height and fresh weight of at least 30 seedlings of each line were measured. For ABA sensitivity assay, 60 seeds were germinated on $1 / 2 \mathrm{MS}$ medium containing different concentrations of $\mathrm{ABA}$ under $28^{\circ} \mathrm{C} / 25^{\circ} \mathrm{C}$ (day/night) with a $12 \mathrm{~h}$ photoperiod. At 6 days after germination, the germination rates and shoot and root lengths for each line were recorded.

\section{Physiological and Biochemical Measurements}

Content of free proline in leaves of rice plants was determined as previously described (Bates et al., 1973). Briefly, leaves were harvested, weighed, and extracted in 3\% sulfosalicylic acid. An aliquot of each extract $(2 \mathrm{~mL})$ was incubated with $2 \mathrm{~mL}$ of ninhydrin reagent $[2.5 \%(\mathrm{w} / \mathrm{v})$ ninhydrin, $60 \%(\mathrm{v} / \mathrm{v})$ glacial acetic acid, and $40 \% 6 \mathrm{M}$ phosphoric acid] and $2 \mathrm{~mL}$ of glacial acetic acid at $100^{\circ} \mathrm{C}$ for $40 \mathrm{~min}$, and the reaction was terminated on ice bath. Four milliliters of toluene solution were added to the reactions, followed by vortex and incubation at $23^{\circ} \mathrm{C}$ for $30 \mathrm{~min}$, followed by measurement of the absorbance at $520 \mathrm{~nm}$. Content of total soluble sugars in leaves of rice plants was measured as previously described (Bailey, 1958). Leaf samples were weighed and then extracted by $80 \%$ ethanol at $80^{\circ} \mathrm{C}$ for $30 \mathrm{~min}$ with occasional agitation. The supernatant was filtered and brought to a final volume of $10 \mathrm{~mL}$ with $80 \%$ ethanol. One milliliter of the extract was incubated with $5 \mathrm{~mL}$ anthrone reagent at $95^{\circ} \mathrm{C}$ for $15 \mathrm{~min}$, and then the reaction was terminated on ice, followed by measurement of the absorbance at $620 \mathrm{~nm}$. Relative water content (RWC) in leaves of rice plants was measured as previously described (Schonfeld et al., 1988). Fully expanded leaves from three- to four-leaf stage soil-grown plants were detached to measure the leaf fresh weight $\left(W_{\mathrm{F}}\right)$, turgid leaf weight $\left(W_{\mathrm{T}}\right)$, and dry weights $\left(W_{\mathrm{D}}\right)$, and $R W C$ were calculated from the equation

$$
R W C(\%)=\left(W_{\mathrm{F}}-W_{\mathrm{D}}\right) /\left(W_{\mathrm{T}}-W_{\mathrm{D}}\right) \times 100 \%
$$

Transpiration rate in leaves was measured using LI-6400 Photosynthesis System (LI-COR, Lincoln, NE, USA). Five flag leaves of 3-month-old plants grown in greenhouse were enclosed in a leaf chamber with a built-in red and blue light source at PAR of $800 \mu \mathrm{mol} \cdot \mathrm{m}^{-2} \cdot \mathrm{s}^{-1}$ in the gas exchange analyzer. The chamber $\mathrm{CO}_{2}$ concentration was controlled at 390 ppm using an automatic $\mathrm{CO}_{2}$ controller in the LI-6400 system. Measurements were performed at 10:00 AM, 12:00 PM, 14:00 PM, and 16:00 $\mathrm{PM}$ in a clear day for each leaf and at least eight measurements for each plant at each time point were collected to calculate the transpiration rate. Content of $\mathrm{Na}^{+}$in root and shoot tissues was analyzed according to a previously described protocol (Jabeen et al., 2014). Briefly, shoots and roots were harvested from at least ten 4-week-old N22oe and WT seedlings grown hydroponically in modified Hogland solution supplemented with or without $150 \mathrm{mM} \mathrm{NaCl}$. Samples were washed three times with deionized water, grinded to fine powder with liquid nitrogen and then dried at $70^{\circ} \mathrm{C}$ for 3 days. After weighting, the dried samples were digested with $10 \mathrm{~mL}$ nitric acid until clarification and the $\mathrm{Na}^{+}$content was measured using a flame atomic absorption spectrometry (AA6300, Shimadzu, Japan). Stomatal closure was examined according to the method described previously (You et al., 2013). Briefly, fully expanded leaves from 8-week-old plants grown under normal or drought stress condition were detached and fixed in $2.5 \%$ glutaraldehyde solution. Stomatal status was monitored by scanning electron microscopy (SU8010, Hitachi, Japan).

\section{Quantification of Endogenous ABA Content}

Leaf tissues (300 mg) were ground in liquid nitrogen. The powder was extracted with $2 \mathrm{~mL} 80 \%$ methanol and kept overnight at $-20^{\circ} \mathrm{C}$, followed by centrifugation at $4^{\circ} \mathrm{C}$ for $10 \mathrm{~min}$ at $12,000 \times \mathrm{g}$. The supernatant was collected, dried under nitrogen gas and dissolved in $0.5 \mathrm{~mL} 2 \%$ ammonia solution. Crude extracts were further purified by Oasis MAX SPE columns (Waters Corp., Milford, MA, USA) that were sequentially preconditioned with $2 \mathrm{~mL}$ methanol and $2 \mathrm{~mL} 2 \%$ ammonia solution. After the supernatant was loaded, the columns were sequentially washed with ammonia solution (2\%) and $2 \mathrm{~mL}$ methanol. ABA was eluted with $4 \mathrm{~mL}$ methanol containing $1 \%$ formic acid. The eluent was dried under nitrogen gas and dissolved in $0.5 \mathrm{~mL} \mathrm{80 \%}$ methanol. Quantification of ABA was performed by a HPLCTriple quadrupole liquid chromatography-mass spectrometry system (Model 1290/6460, Agilent Technologies, Santa Clara, CA, USA) with stable isotope-labeled ABA as a standard according to a previously described method (Fu et al., 2012).

\section{qRT-PCR for Gene Expression Analysis}

Total RNA was extracted from frozen leaf samples using TRIzol reagent (Invitrogen, Shanghai, China) and then treated with RNase-free DNase (TaKaRa, Dalian, China). First-strand cDNA was synthesized from $1 \mu \mathrm{g}$ of total RNA using AMV reverse transcriptase (TaKaRa, Dalian, China) according to the manufacturer's recommendation. qRT-PCR was performed on a CFX96 real-time PCR system (Bio-Rad, Hercules, CA, USA) using Fast Essential DNA Green Master kit (Roche Diagnostics, Shanghai, China). Each qRT-PCR reaction contained $12.5 \mu \mathrm{L} \mathrm{2x}$ Fast essential buffer (Roche Diagnostics, Shanghai, China), $1 \mu \mathrm{g}$ cDNA and $10 \mu \mathrm{moL}$ of each of gene-specific primers in a final volume of $25 \mu \mathrm{L}$. A rice Actin gene was used as an internal control to normalize the data and relative expression levels of genes of interest were calculated using the $2^{-\Delta \Delta C T}$ method. Gene-specific primers used in qRT-PCR are listed in Supplementary Table S1.

\section{Microarray Analyses of Gene Expression Profiling}

Leaf samples were collected from 3-week-old N22oe and WT plants and microarray analyses were performed using Affymetrix 
Rice Genome Array by standard protocol. All procedures for total RNA extraction, probe preparation, hybridization, scanning, data collection, and bioinformatics analyses were carried out at Beijing Capitalbio Biotechnology Company Ltd (Beijing, China). Genes with a twofold change in the expression level between N22oe and WT plants were defined as differentially expressed genes.

\section{Statistical Analysis}

All experiments were repeated independently for at least three times and data were subjected to statistical analysis using the Student's $t$-test at $p=0.05$ level.

\section{RESULTS}

\section{ONAC022 is a Stress-Responsive NAC Gene in Rice}

The ONAC022 gene encodes a 316 aa protein with a typical NAC domain, which can be divided into five subdomains, namely A, B, C, D, and E (Ooka et al., 2003) (Figure 1A). In addition, the $\mathrm{C}$-terminal region of the ONAC022 protein also contains newly identified unique conserved $\mathrm{C} 1$ and $\mathrm{C} 2$ domains that are absent in other NAC proteins (Kato et al., 2010) (Figure 1A). Phylogenetic analysis suggests that ONAC022 belongs to Group B (Nuruzzaman et al., 2010) or Phylogeny Group IV (Fang et al., 2008), which contains 14 members (Figure 1B). Phylogenetically, ONAC022 is closely related to rice ONAC095 and Arabidopsis ANAC036, showing 62 and 52\% of identity at amino acid level, respectively, but is less related to other known stress-responsive NAC proteins in rice (Figure 1B). Bioinformatics analysis indicates that the promoter region $(1.5 \mathrm{~Kb}$ upstream of the start codon) of the ONAC022 gene contains some stress responserelated cis-elements, including one ABRE element, two MYB binding sites, two TCA elements, one TC-rich element and one GCC box (Figure 1C). To explore the possible involvement of ONAC022 in abiotic stress response, we analyzed the expression patterns of ONACO22 in rice plants after treatment with abiotic stresses and ABA. qRT-PCR analyses revealed that the expression of ONAC022 was significantly and rapidly induced within $2 \mathrm{~h}$ after salt and drought treatment, leading to fourfold to sixfold of increases (Figures 2A,B). Treatment of rice plants with $\mathrm{ABA}$ induced the expression of ONAC022, showing threefold to fourfold of increase during 6-12 h after treatment (Figure 2C). These data indicate that ONACO22 is a stress-responsive NAC gene in rice.

\section{ONAC022 is a Nucleus-Localized Transcriptional Activator}

To examine the subcellular localization of ONAC022, agrobacteria carrying pFGC-EGFP:ONAC022 and pFGCEGFP (as a negative control) were infiltrated into leaves of 4-week-old Nicotiana benthamiana plants that expressed a red nuclear marker RFP-H2B protein (Chakrabarty et al., 2007). Confocal micrographs showed that the GFP:ONAC022 fusion protein was solely and clearly localized to the nucleus, co-localized with the known nucleus marker RFP-H2B protein
(Figure 3A), whereas the GFP alone distributed ubiquitously throughout the cell without specific compartmental localization (Figure 3A). These data demonstrate that the ONAC022 protein is localized to nucleus of the cells.

To examine whether the ONAC022 protein had DNA binding activity, GST-ONAC022 fusion protein was purified to homogeneity in native PAGE. EMSA results revealed that the GST-ONAC022 protein bound to the biotin-labeled wNACRS fragment, which contained the CATGTG and CACG core motifs (Xie et al., 2000; Trans et al., 2004), forming a specific DNAprotein complex, but did not bind to the biotin-labeled mNACRS fragment (Figure 3B), in which the CATGTG and CACG motifs were replaced with the sequences AAAAAA and AAAA, respectively. In the competition binding assay, binding of GSTONAC022 to the labeled wNACRS fragment was attenuated in the presence of excess unlabeled wNACRS fragment in the reaction but was not affected by unlabeled mNACRS fragment (Figure 3B). The purified GST protein did not bind to the wNACRS fragment (Figure 3B). These results indicate that the ONAC022 protein could specifically bind to the cis-element NACRS of the NAC proteins.

To determine whether ONAC022 had transcriptional activator activity, the full ONAC022 protein, an N-terminal fragment ONAC022 $\Delta \mathrm{C}$ (lacking 151-316 aa at C-terminal) and a C-terminal fragment ONAC022 $\Delta \mathrm{N}$ (lacking 1-150 aa at $\mathrm{N}$-terminal) were each fused to the GAL4 DNA-binding domain of the $\mathrm{pBD}$ vector (Figure 3C). All yeast transformants grew well in SD/Trp ${ }^{-}$medium (Figure 3C). On SD/Trp ${ }^{-} \mathrm{His}^{-}$ medium, only transformants carrying pBD-ONAC022 or $\mathrm{pBD}-\mathrm{ONAC} 022 \Delta \mathrm{N}$ grew and showed $\beta$-galactosidase activity, whereas transformants carrying $\mathrm{pBD}-\mathrm{ONAC} 022 \Delta \mathrm{C}$ and $\mathrm{pBD}$ empty vector did not (Figure 3C). These results indicate that the ONAC022 protein is a transcriptional activator and that the C-terminal region of ONAC022 is required for its transactivation activity.

\section{Generation of the ONAC022-Overexpressing (N220e) Transgenic Rice}

The ONAC022 gene was transformed into rice cv. Xiushui134 under the control of the maize ubiquitin promoter (Figure 4A). A total of 23 independent transgenic lines were generated. After screening by Hgr resistance phenotype on 1/2 MS medium, three independent transgenic lines (N22oe-33, 34, and 37) were selected as candidates of single copy lines and were confirmed by Southern blotting with a fragment of the Hgr gene as a probe. Because there is no EcoRI sites in the region between $\mathrm{LB}$ and $\mathrm{RB}$ in the pCoUm vector, a single band of the EcoRIdigested genomic DNA from each of these three lines was detected (Figure 4B), indicating that the transgenic lines N22oe33,34 , and 37 contained a single copy of the transgene. qRT-PCR analysis showed that the ONAC022 gene was expressed normally in the transgenic lines (T3 generation) and the expression levels in N22oe-33, 34, and 37 lines were 27, 22, and 11 times higher than that in WT plants, respectively (Figure 4C). During the experiments under normal watered condition in 
A ONAC022 ---MAAAVAVAGPSLEVEODLF-GFRFP TEEELLDFYLSRWLGKKLHFNIIGTLNIYRHDPWD LPGMAIIGEREWYFF 76 ONAC095 MEQORSRSTAAGGEVEVEO-LP-GFRFHP TEE ELLEFYLKOWDGKKLKF DIIPTYHL YRHDPRE LPGLARI GEREWYFF 78

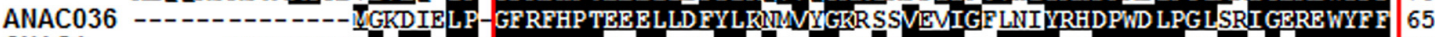

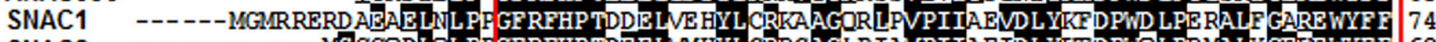
SNAC2 -------1SGGQDLOLPPGFRFHPTDEELMHYLCRRCAGLP IAVPIIAE IDLYKFDPWDLPRMALYGEKE WYFE 68

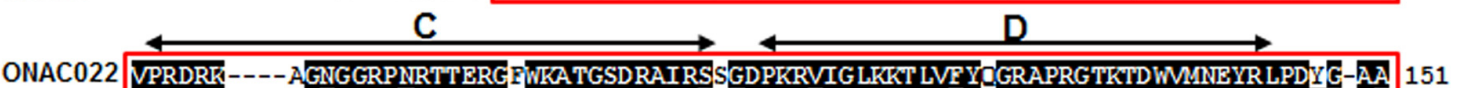
ONAC095 VPRDRKOATGGGGGGRP SRTTERG FWKATGSDRAIRCAADPKRLIG LKKT LVYYEGRAPRGTKTDWMMNEYRLPDAA-AI 157 ANAC036 VPRERK----HGNGGRP SRTTEKG YWKA TGSDRI II ISLS EPKRVIG LKKT LVF YRGRAP GG SKTDWMMNEFRMPDN---137 SNAC1 TPRDRK----YPNG SRPNRAAGNGYWKA TGADKRVAPRG---RTLG IKKKALVF YAGKAPRG VKTDWIMABEYR LADAGRAA 147

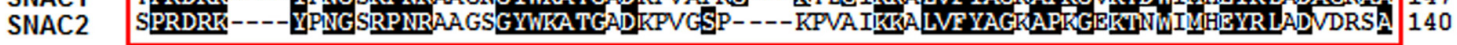

$$
\stackrel{\mathrm{C} 1}{\stackrel{\mathrm{E} \longrightarrow}{\rightleftarrows}}
$$

ONAC022 BAAAAPP--PKEDYVLCKIYRKATP LKELEQRASAMEEMORGSSHGD YTATRAS LVHDASASTTEDD YFS SDDVHDSGELIZ 229

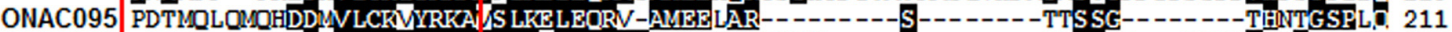
ANAC036 -CS LP-----KDWVLCKIYRKAITS LKMLEORAEME AKMNOTC PN-----S------- PLSSSE---T ISFVGKEEMMTT 195

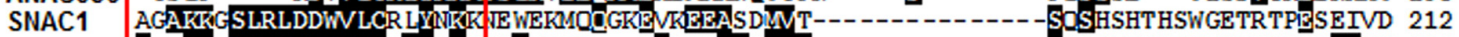

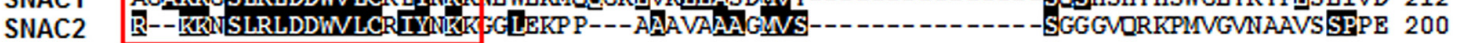
C2

ONAC022 SSSSSAAPSGSSSKN--- GGAGAPREAKKKEEADVTVTAASAT SLOLPAVSOLF SLOLP----AMDW̄LDPFLTC----- 296 ONAC095 ODSSSI SISSSSD-------AMKKKWWGDEASAAAHELVRPATLSLPQLEVARPOSGLWWOEPFLTC----- 273 ANAC036 SERAPOVIAMEEANK---IOMHOENAKT EEKOREAETKE PSS SLKLPFG-SLPELOLPKP- - GVEW--DOLLS------ 260 SNAC1 NDPFPE LDSF PAFOP--APPPATAIMNPEKRSRDDATAAAAAAAATIPRNNSSLFVDLSYDD IOGMYSG LDMLPPGDDFYS 290 SNAC2 QKPWAGGAE PD LAAYYDRPSDSMPRLHAD SSCSEQVLS PEFACEVQSOPKISEWERTFATVGPINPAASILDPAG---S 277

ONAC022 ---LRSPWODOHCLS PYAHLLYY

ONAC095 --LRSPWRETWSP-YYASVLNF

ANAC036 ---ISPWLONLTP--IVNIYW

SNAC1 SLFASPRVKGTTPRAGAGMG--INPF

SNAC2 GGLGGLGGGGSDPLLDD ILWWGKPF

B

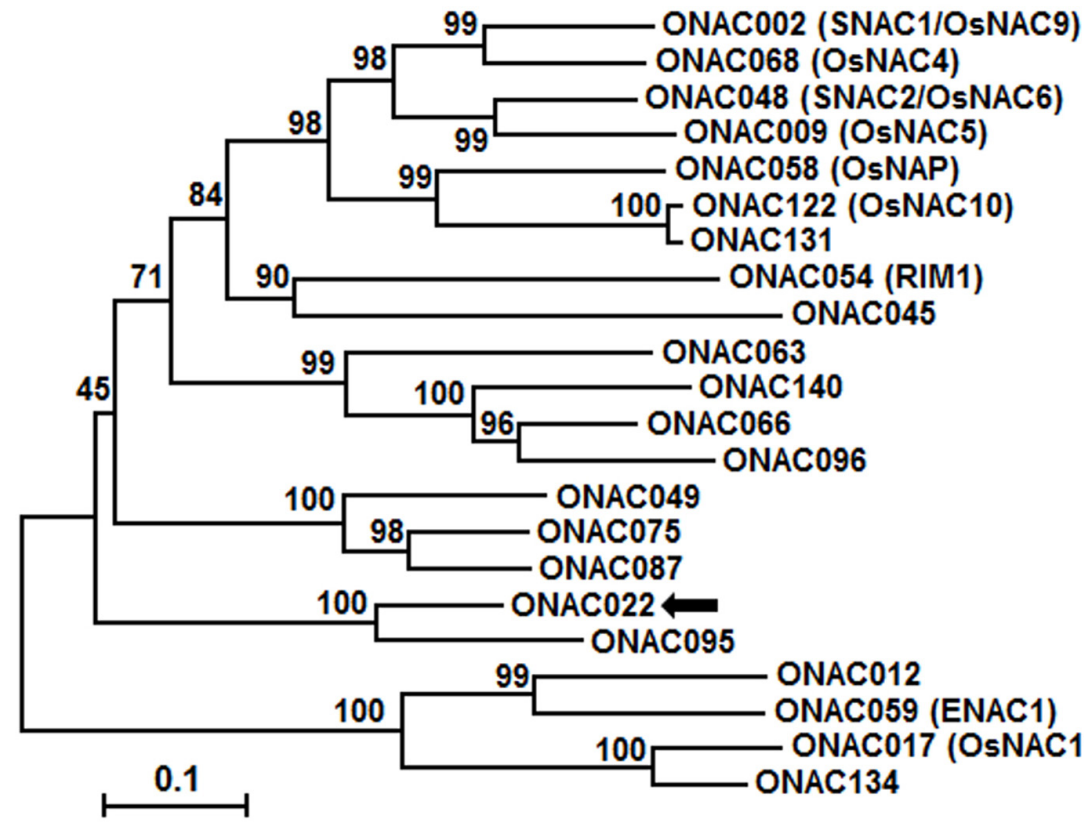

Drought, salt Drought, cell death Drought, salt, disease Drought, salt Drought, salt Drought, disease Disease Disease Drought, salt

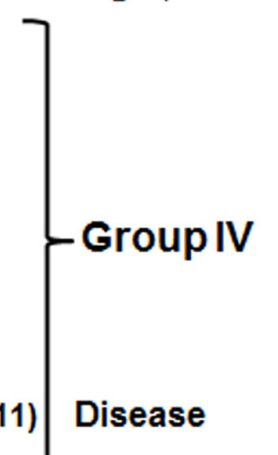

c
$-1.5 \mathrm{~Kb}$ مQ $\nabla$
$\nabla<\diamond$

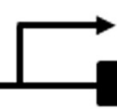
ATG

\section{OTCA element $\quad \nabla$ MYB binding site $\bigcirc$ ABRE $\diamond$ TC rich $\diamond$ GCC box}




\section{FIGURE 1 | Continued}

Characterization of rice ONAC022 protein and gene. (A) Alignment of ONAC022 with rice ONAC095, SNAC1 (OsNAC9), and SNAC2 (OsNAC6) and Arabidopsis ANAC036. Identical amino acids are shaded in black and the conserved NAC domain is boxed. Black arrowed lines indicate the locations of the five highly conserved subdomains A-E, while red arrowed lines show the locations of the newly identified C1 and C2 domains. (B) Phylogenetic tree analysis of ONAC022 with other known stress-responsive rice NAC proteins. Sequence alignment was performed using Clustal X1.81 program and phylogenic tree was created and visualized using MEGA 5.05. Protein sequences used for alignment are as follow: ONAC012 (Os05g37080), ONAC022 (Os03g04070), ONAC017 (Os11g05614), ONAC049 (Os08g02160), ONAC059/ENAC1 (Os01g64310), ONAC063 (Os08g33910), ONAC066 (Os03g56580), ONAC075 (Os01g66490), ONAC087 (Os05g34600), ONAC095 (Os06g51070), ONAC096 (Os07g04560), ONAC134 (Os12g05990), ONAC140 (Os12g43530), ONAC002/SNAC1/OsNAC9 (Os03g60080), ONAC048/SNAC2/OsNAC6 (Os01g66120), ONAC058/OsNAP (Os03g21060), ONAC122/OsNAC10 (Os11g03300), ONAC131 (Os12g03040), ONAC068/OsNAC4 (Os01g60020), ONAC009/OsNAC5 (Os11g08210), ONAC054/RIM1 (Os03g02800), ONAC045 (Os11g03370). Reported names and functions in stress response of the known stress-responsive NAC genes were given in parentheses in the tree and listed at right of the tree, respectively. (C) Distribution of major stress-related cis-elements in the promoter region of the ONACO22 gene.

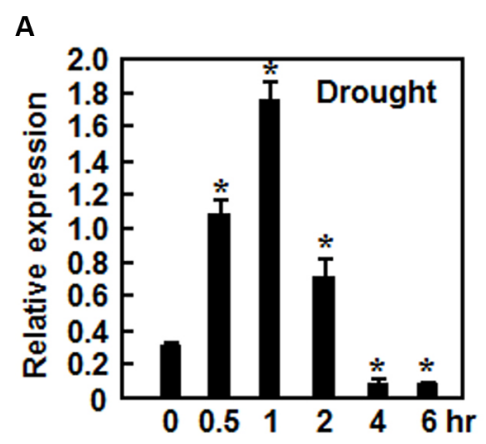

B

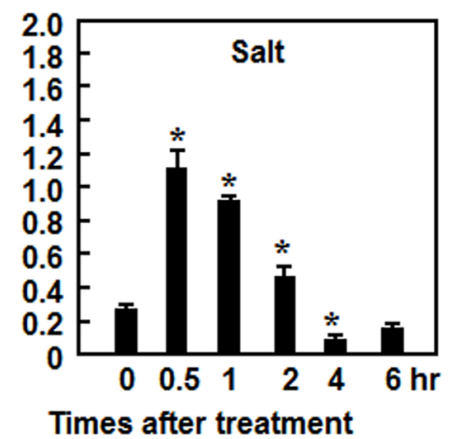

C

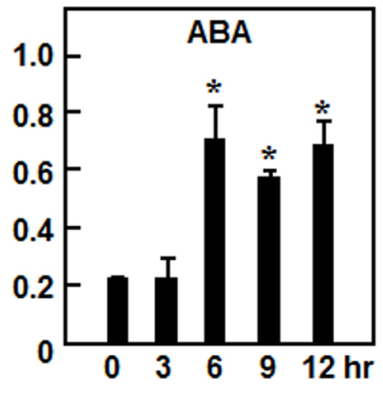

FIGURE 2 | Induction of ONAC022 expression by drought, salt, and abscisic acid (ABA). Two-week-old seedlings were exposed to drought (A), 150 mM $\mathrm{NaCl}(\mathbf{B})$, or treated by foliar spraying with $100 \mu \mathrm{M} \mathrm{ABA}(\mathbf{C})$ and leaf samples were collected at indicated time points for analyses of gene expression by qRT-PCR. Relative expression levels as compared to those of the actin gene at each time point are presented as the means \pm SD from three independent experiments. ${ }^{*}$ Above the columns indicate significant differences at $p \leq 0.05$ level with corresponding controls.

greenhouse, we noticed that the N22oe plants showed growth retardation (Figure 4D), leading to $25-29 \%$ of reduction in plant height (Figure 4E), as compared to WT plants. In addition, we also noted that the panicles of the N22oe plants grown under greenhouse condition were smaller than the WT plants (Figure 4F), leading to significant reductions in grain numbers per panicle and 1000-grain weight (Figures 4G,H). These data indicate that overexpression of ONAC022 has a negative effect on growth and a penalty on grain yield in transgenic rice. No significant difference in other morphological and developmental characters such as tiller number, flowering and heading was observed between the N22oe and WT plants.

\section{Increased Drought and Salt Tolerance in N22oe Plants}

To examine whether ONAC022 plays a role in abiotic stress tolerance, we compared the drought and salt tolerance of the N22oe and WT plants at vegetative growth stage. Before drought stress treatment, the N22oe and WT seedlings exhibited similar growth status (Figure 5A). The WT seedlings started to show leaf rolling at 10 days (Figure 5B) and became severe leaf rolling and wilting at 12 days after withholding water (Figure 5C). During the drought stress process, however, the N22oe seedlings showed delayed and less leaf rolling and did not show wilting symptom during the drought stress process, as compared with the WT seedlings (Figures 5B,C). After re-watering regularly for 12 days, only $24 \%$ of the WT seedlings were recovered, while 85 , 78 , and 66 of the N22oe-33, 34, and 37, respectively, survived (Figures 5D,E). Similarly, the fresh weights of individual N22oe plant were significantly higher than WT at 12 days after recovery, giving approximately $50 \%$ of increase (Figure 5F). These data demonstrate that the N22oe plants exhibit increased tolerance to drought stress and that ONAC022 plays a critical role in rice drought stress tolerance.

We next compared the salt tolerance of the N22oe and WT plants. No significant difference between the N22oe and WT lines was observed for seed germination and seedling growth on 1/2 MS medium without supplement of $\mathrm{NaCl}$ (Figure 6A, upper). However, in the presence of $150 \mathrm{mM} \mathrm{NaCl}$, seed germination and seedling growth of the WT were markedly inhibited at 7 days after planting on 1/2 MS medium. By contrast, seeds of the N22oe-33, 34, and 37 lines germinated and the seedlings grew normally on $1 / 2 \mathrm{MS}$ medium supplemented with $150 \mathrm{mM}$ $\mathrm{NaCl}$ (Figure 6A, lower) although the seedling growth of the N22oe lines was inhibited to some extent as compared with those grown on $\mathrm{NaCl}$-free medium (Figure 6A). The shoot length of the N22oe seedlings was similar to that of the WT seedlings when grown under normal condition, but was significantly higher than the WT seedlings when grown under $\mathrm{NaCl}$ stress, leading to twofold to threefold of increases (Figure 6B). The root length of the N22oe seedlings was also higher than that 


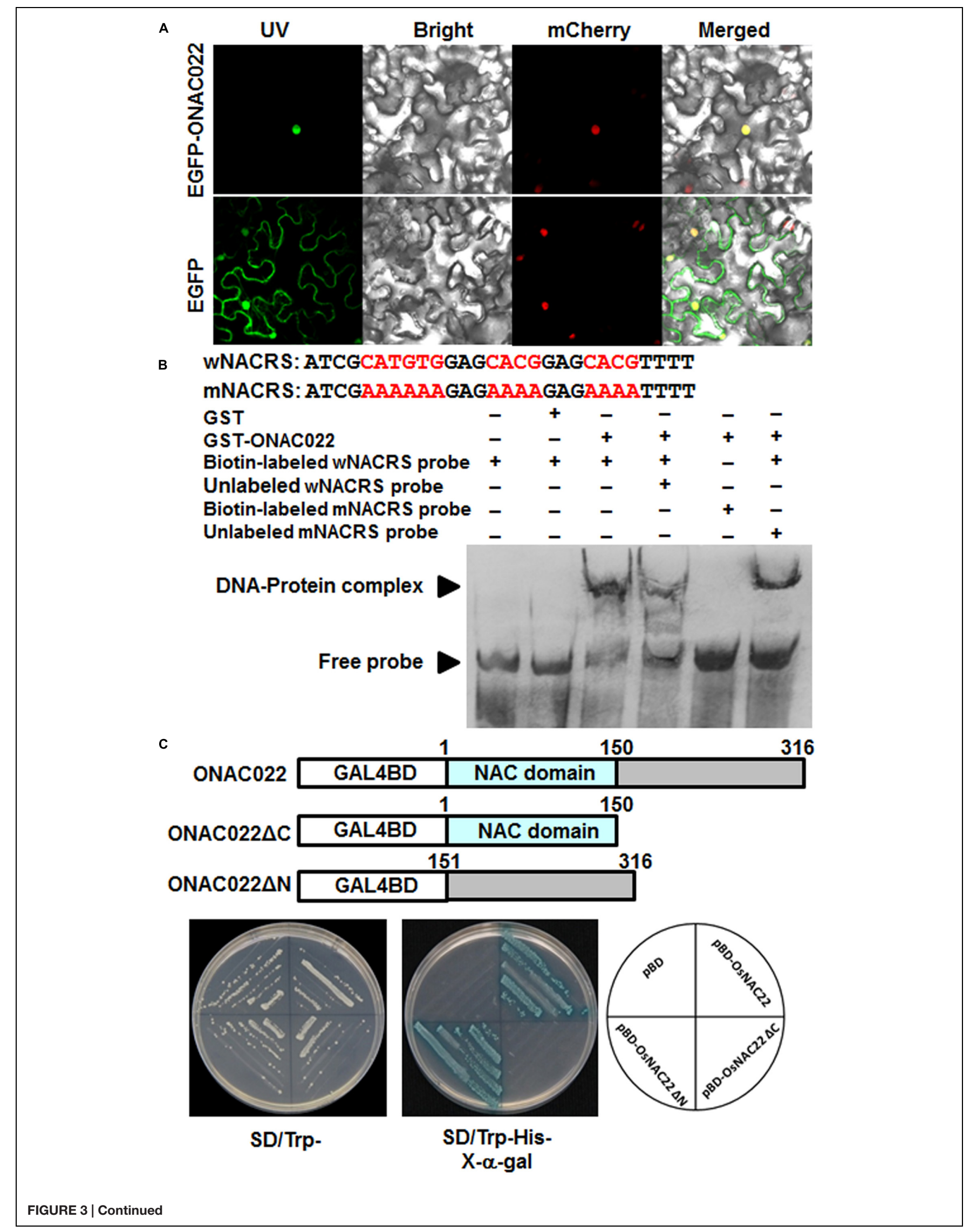




\section{FIGURE 3 | Continued}

Subcellular localization and transactivation activity of the ONAC022 protein. (A) ONAC022 is localized in nucleus. Agrobacteria carrying

pFGC-Egfp-ONAC022 or pFGC-Egfp empty vector were infiltrated into leaves of Nicotiana benthamiana plants expressing a red nucleus marker protein RFP-H2B and leaf samples were collected at $24 \mathrm{~h}$ after infiltration for observation under a confocal laser scanning microscope. Images were taken in dark field for green fluorescence (left) and red fluorescence (middle right), white field for cell morphology (middle left) and in combination (right), respectively. (B) DNA binding activity of ONAC022. Wild type version of the cis-element sequence (WNACRS) and a mutated version (mNACRS) were used. Electrophoretic mobility shift assays were performed using the recombinant GST-fused ONAC022 protein. Biotin-labeled wNACRS and mNACRS probe and biotin-labeled wNACRS probe in combination with unlabeled wNACRS or mNACRS probe were incubated with GST-fused ONAC022 protein or a purified GST preparation as a negative control. Specific DNA-protein complexes and free probes are indicated by the arrowheads on left. (C) ONAC022 has transactivation activity. Yeast cells carrying pBD-ONAC022,

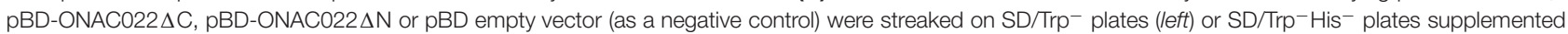
with $\mathrm{x}$ - $\alpha$-gal (right) for 3 days at $28^{\circ} \mathrm{C}$.

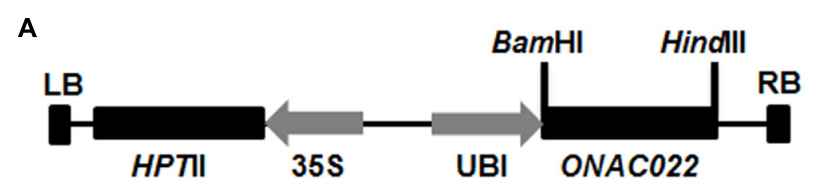

B

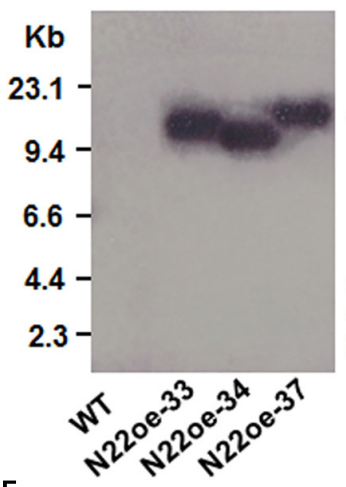

F

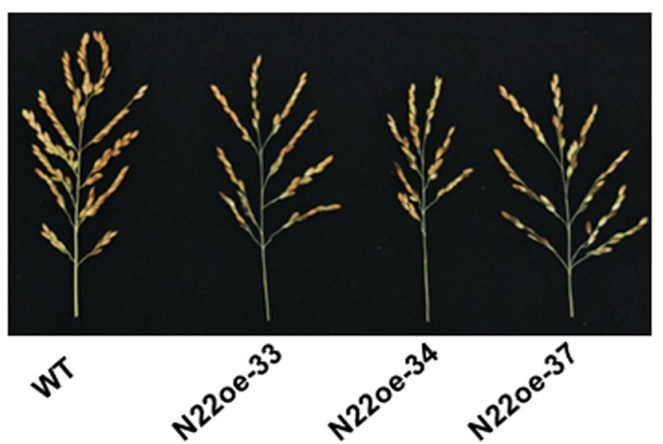

C

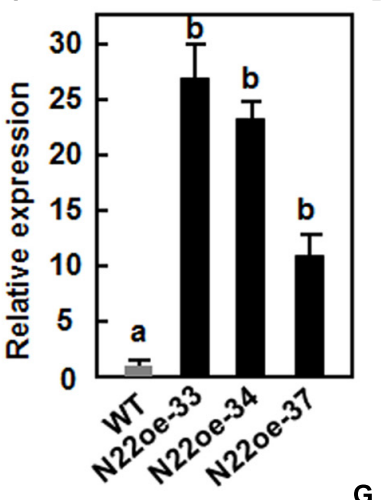

G

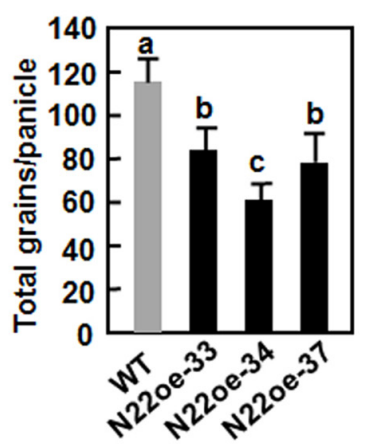

E
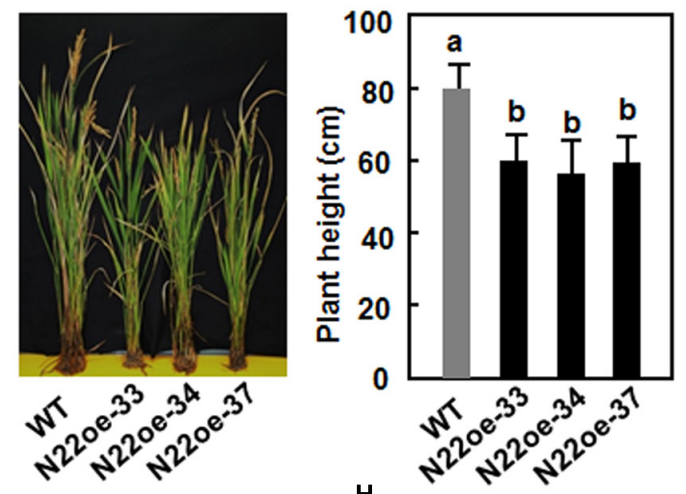

H

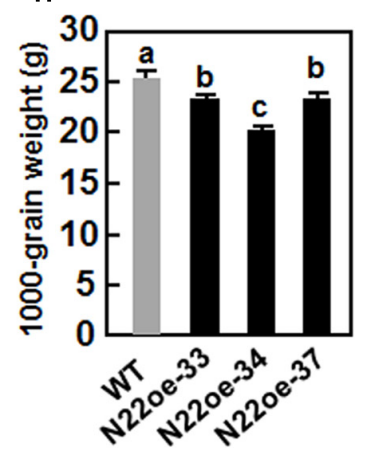

FIGURE 4 | Characterization of the ONAC022-overexpressing transgenic rice lines and their agronomic traits. (A) Schematic diagram of the overexpression construct used for rice transformation. Hptll, hygromycin (Hgr) phosphotransferase II; LB, left border; RB, right border; Ubi, maize ubiquitin promoter; 35S, CaMV 35S promoter. (B) Southern blot analysis of copy number of the transgene in T4 generation of the N22oe lines. Genomic DNA ( $50 \mu \mathrm{g}$ ) extracted from the N22oe and wild-type (WT) plants was digested with EcoRI and probed with a DIG-labeled fragment of the Hptll gene. (C) Expression levels of the ONACO22 gene in the N22oe lines. (D) Growth phenotypes of the N22oe plants at heading stage under normal watered condition in greenhouse. (E) Reduced plant height of the N22oe plants grown under normal watered condition in greenhouse. (F) Comparison of the panicles between the N22oe and WT plants grown under normal watered condition in greenhouse. (G) Numbers of grains per panicle between the N22oe and WT plants grown under normal watered condition in greenhouse. (H) Weights of 1000-grain from the N22oe and WT plants grown under normal watered condition in greenhouse. Data presented $(\mathbf{C}, \mathbf{E}, \mathbf{G}, \mathbf{H})$ are the mean \pm SD from three independent experiments and different letters above the columns indicate significant differences at $p \leq 0.05$ level with corresponding WT.

of the WT seedlings when grown under normal condition or $\mathrm{NaCl}$ stress and showed 70-85\% increase over the WT seedlings when grown under $\mathrm{NaCl}$ stress condition (Figure 6C). Also, the lateral root numbers of the N22oe seedlings were much more than that of the WT seedlings when grown under $\mathrm{NaCl}$ stress condition, leading to 1.5 - to 3 -fold of increases (Figure 6D). We also measured the $\mathrm{Na}^{+}$content in shoots and roots of WT and N22oe seedlings grown under normal or salt stress condition. When grown in normal Hogland solution, the $\mathrm{Na}^{+}$ content in roots and shoots showed no significant difference 

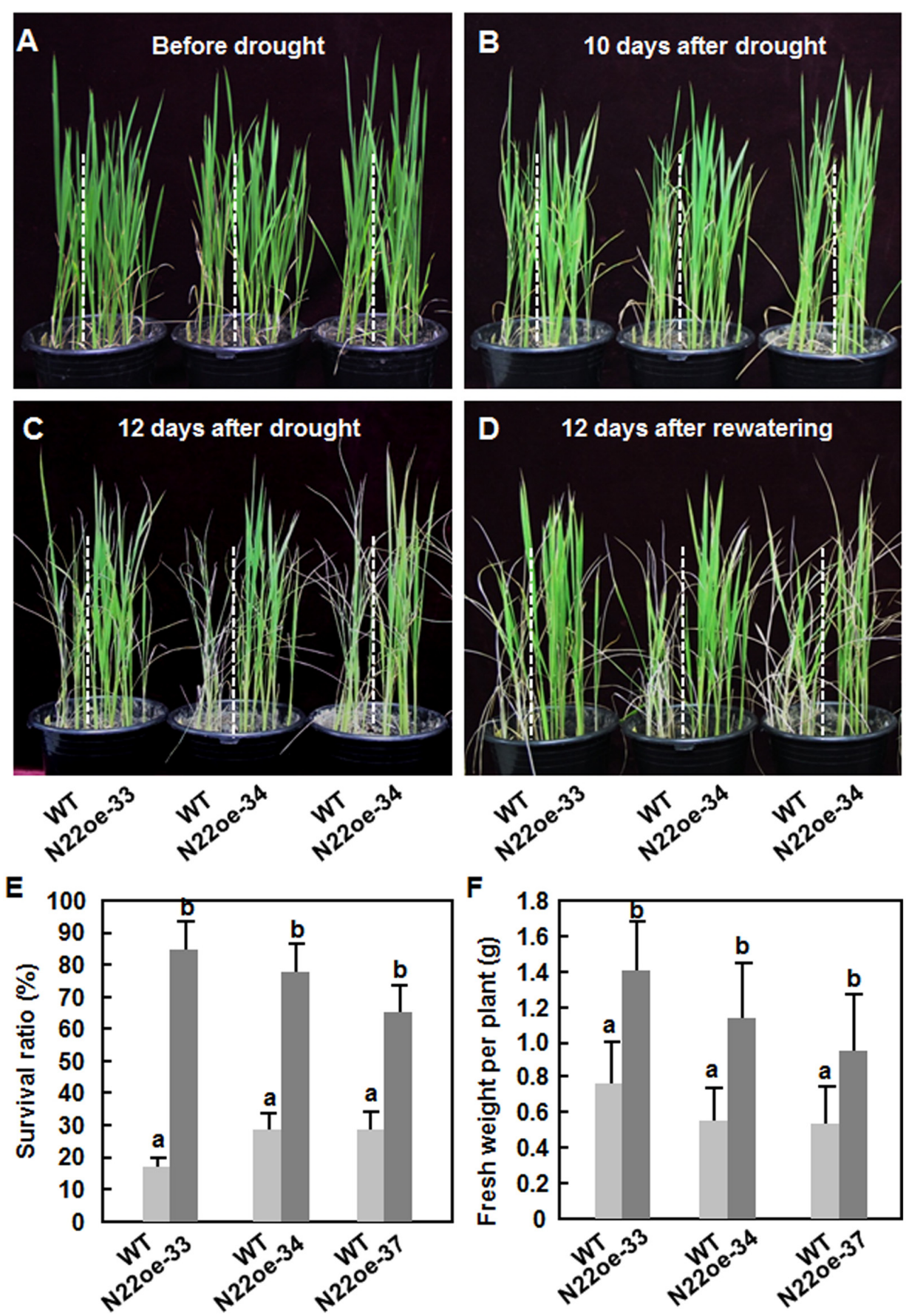

FIGURE 5 | Increased drought tolerance in N22oe plants. (A-D) Phenotype of the N22oe and WT seedlings at different stages during the drought stress experiments. The N22oe seedlings were grown in barrels each with WT seedlings as a control. (E) Survival ratios of the N22oe and WT plants at 12 days after re-watering. (F) Growth biomass of the seedlings after drought stress treatment. Data presented in (E,F) are the mean \pm SD from three independent experiments and different letters above the columns indicate significant differences at $p \leq 0.05$ level.

between WT and N22oe seedlings (Figures 6E,F). The $\mathrm{Na}^{+}$ contents in roots and shoots of plants grown in Hogland solution supplemented with $150 \mathrm{mM} \mathrm{NaCl}$ were significantly increased as compared to those in plants grown normal Hogland solution (Figure 6E). However, the $\mathrm{Na}^{+}$content in roots and shoots of the N22oe plants were significantly reduced by $25-55$ and $32-47$, respectively, as compared to those in WT plants (Figures 6E,F). These results suggest that the N22oe plants show increased salt tolerance and that ONAC022 functions importantly in rice salt tolerance. 


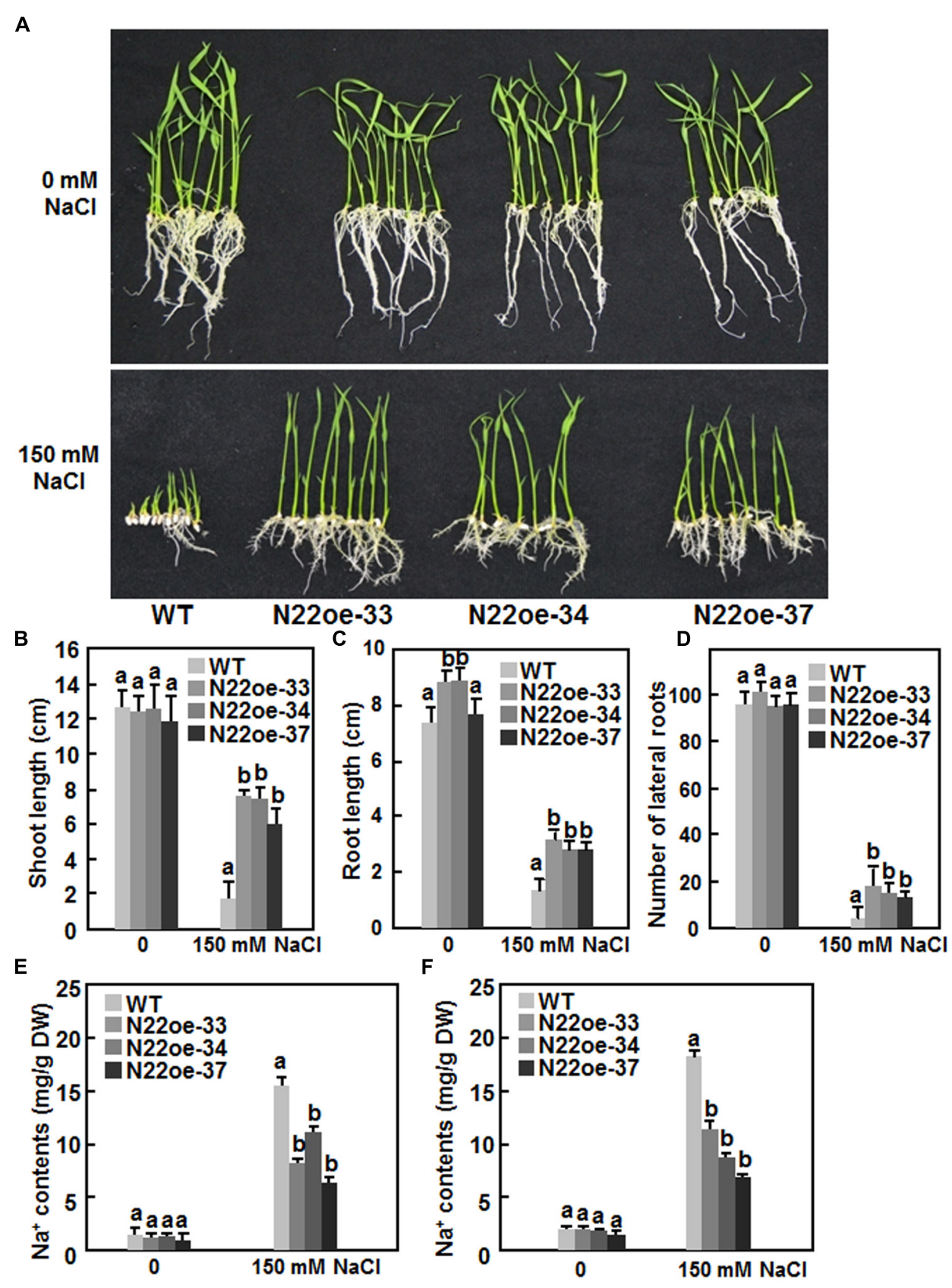

FIGURE 6 | Increased salt tolerance in N22oe plants. (A) Growth performance of the N22oe and WT seedlings grown on 1/2 MS medium supplemented with or without $150 \mathrm{mM} \mathrm{NaCl}$ (B,C) Shoot and root length of the N22oe and WT seedlings grown on 1/2 MS medium supplemented with or without 150 mM NaCl.

(D) Number of lateral roots of the N22oe and WT seedlings grown on 1/2 MS medium supplemented with or without $150 \mathrm{mM}$ NaCl. (E,F) Na+ contents in roots and shoots of the N22oe and WT seedlings grown in modified Hogland solution with or without $150 \mathrm{mM} \mathrm{NaCl}$. Data presented in (B-F) are the mean \pm SD from three independent experiments and different letters above the columns indicate significant differences at $p \leq 0.05$ level with corresponding WT.

\section{Increased Contents of Stress-Related Metabolites and Reduced Transpiration in N22oe Plants}

To explore the possible physiological mechanism responsible for the increased drought and salt tolerance in N22oe plants, we compared some stress-related physiological changes between the N22oe and WT plants grown under normal and drought conditions. Under normal growth condition, the proline contents in the N22oe plants were markedly higher than the WT plants, leading to $20-78 \%$ of increases (Figure 7A), whereas no significant difference in the contents of soluble sugars was observed between the N22oe and WT plants (Figure 7B). At 10 days after drought stress treatment, the proline and soluble 
sugar contents were increased significantly both in N22oe and WT plants as compared with those in plants grown under normal condition (Figures 7A,B). However, the increases in proline and soluble sugar contents in N22oe plants were much evident than those in WT plants under drought stress condition, resulting in $32-73$ and $55-110 \%$ of increases for the proline and soluble sugar contents, respectively (Figures 7A,B). The rates of water loss in detached leaves of the N22oe plants were approximately $8-15 \%$ lower than those in detached leaves of the WT plants during a period of $3-7.5 \mathrm{~h}$ after detachment (Figure 7C). Similarly, the transpiration rates in leaves of the N22oe plants under normal sunlight condition were reduced by $0.5-1.17 \%$, as compared with those in WT plants, at 12:00, 14:00, and 16:00 (Figure 7D). Furthermore, we also examined the stomatal density and behavior between the N22oe and WT plants grown under normal and drought stress conditions. No difference in stomatal density was observed (Figure 7E) between the N22oe and WT plants, but the percentages of open stomata in leaves of the N22oe plants grown under drought condition were significantly lower than that in the WT plants (Figure 7F). These results indicate that increased contents of stress-related metabolites, reduced transpiration rates and less open stomata may be the causes that confer the increased drought tolerance in the N22oe plants.

\section{Increased ABA Sensitivity in N22oe Plants}

The ABA sensitivity of the N22oe lines was examined and compared with WT by analyses of seed germination and seedling growth. On 1/2 MS medium without ABA, seeds of the N22oe and WT lines germinated normally and no significant difference was observed between the N22oe and WT lines (Figures 8A,B). However, on $1 / 2 \mathrm{MS}$ medium supplemented with $3 \mu \mathrm{M}$ or $6 \mu \mathrm{M}$ $\mathrm{ABA}$, germination of seeds of the N22oe lines was significantly inhibited as compared with the WT line (Figures 8A,B), resulting in $35-54 \%$ of reduction in seed germination rate as compared with the WT at the same ABA concentration (Figure 8B). Similarly, the N22oe seedlings grew better than those of the WT seedlings on 1/2 MS medium without ABA (Figure $8 \mathrm{C}$ ) and had longer shoots and roots, leading to $17-27$ and $11-23 \%$ of increases for shoot and root lengths (Figures 8D,E). However, significant growth inhibition of the N22oe seedlings grown on $1 / 2$ MS medium with $3 \mu \mathrm{M}$ or $6 \mu \mathrm{M}$ ABA was observed (Figure 8C), resulting in $45-52$ and $67-85 \%$ of reduction for shoot length at $3 \mu \mathrm{M}$ or $6 \mu \mathrm{M}$ ABA and $66-72$ and $83-93 \%$ of decrease for root length at $3 \mu \mathrm{M}$ or $6 \mu \mathrm{M}$ ABA, respectively, as compared with the WT seedlings (Figures 8D,E). These data indicate that overexpression of ONACO22 results in increased ABA sensitivity of the transgenic rice.

\section{Increased ABA Biosynthesis and Contents in N22oe Plants}

The increased ABA sensitivity in the N22oe plants led us to examine whether overexpression of ONAC022 affects the endogenous level of $\mathrm{ABA}$ and its biosynthesis in transgenic rice. As shown in Figure 9A, the endogenous $\mathrm{ABA}$ content in the N22oe-33 and 37 plants was significantly higher than that in the WT plants, leading to 60 and $46 \%$ of increases, respectively. Meanwhile, the expression of several genes involved in ABA biosynthesis such as OsNCED1, OsNCED3, OsNCED4, OsNCED5, and OsPSY was upregulated in the N22oe plants grown under normal condition, resulting in increases of 2.8to 14.9-fold for OsNCEDs and 1.7- to 2.6-fold for OsPSY over those in WT plants (Figure 9B). These results suggest that overexpression of ONAC022 can upregulate the expression of many ABA biosynthesis-related genes and thus increase endogenous ABA content in the N22oe plants.

\section{Upregulated Expression of ABA- and Stress-Responsive Genes in N22oe Plants}

To gain further insights into the mechanism of the increased drought and salt tolerance in N22oe plants, the gene expression profiles between 3-week-old N22oe and WT plants grown under normal condition were determined and compared using the Affymetrix rice gene chip. A total of 1059 genes were identified as differentially expressed genes (486 up-regulated and 573 downregulated) that showed a $>$ twofold change in the transcript levels in N22oe plants (Supplementary Figure S1), compared with the transcript levels in WT plants, including a number of stress-responsive genes encoding for protein phosphatase 2Cs (LOC_Os11g01790 and LOC_Os12g01770), late embryogenesis abundant proteins (LOC_Os03g20680 and LOC_Os01g50910) and bZIP protein (LOC_Os02g14910; Supplementary Table S2). Furthermore, we selected 11 stress-responsive genes and compared their expression in the N22oe and WT plants grown under normal conditions. As shown in Figure 9C, the expression levels of OsPP2C06/OsABI2, OsPP2C49, and OsPP2C68, three members of the PP2C family known to be involved in abiotic stress tolerance (Singh et al., 2010), were up-regulated in the N22oe plants, showing increases of 1.3- to 10.5-fold over those in WT plants. Several stress-related TF genes such as OsbZIP23 (Xiang et al., 2008), OsDREB2a (Dubouzet et al., 2003), OsMYB2 (Yang et al., 2012), and OsAP37 (Oh et al., 2009) were up-regulated in the N22oe plants, giving increases of 0.34- to 1.51-fold over those in WT plants (Figure 9C). Furthermore, the expression levels of some stress-related genes like OsRAB21 (Xiong et al., 2014), OsLEA3 (Xiao et al., 2007), OsERD1 (a homolog of Arabidopsis AtERD1; Kiyosue et al., 1994), and OsP5CS1 (Xiong et al., 2014) in N22oe plants were also upregulated with increases of 0.3 - to 1.4-fold for OsERD1 and OsP5CS1 and 14- to 35-fold for OsRAB21 and OsLEA3 over those in WT plants (Figure 9C). These data indicate that overexpression of ONACO22 in N22oe plants, affects the expression of a set of stress-related genes and thus confers an increased drought and salt tolerance in transgenic rice.

\section{DISCUSSION}

The NAC TFs represent a quite large family with 151 members in rice (Fang et al., 2008; Nuruzzaman et al., 2010). Although at least 10 rice NAC genes such as ONAC002 (SNAC1/OsNAC9; Hu 

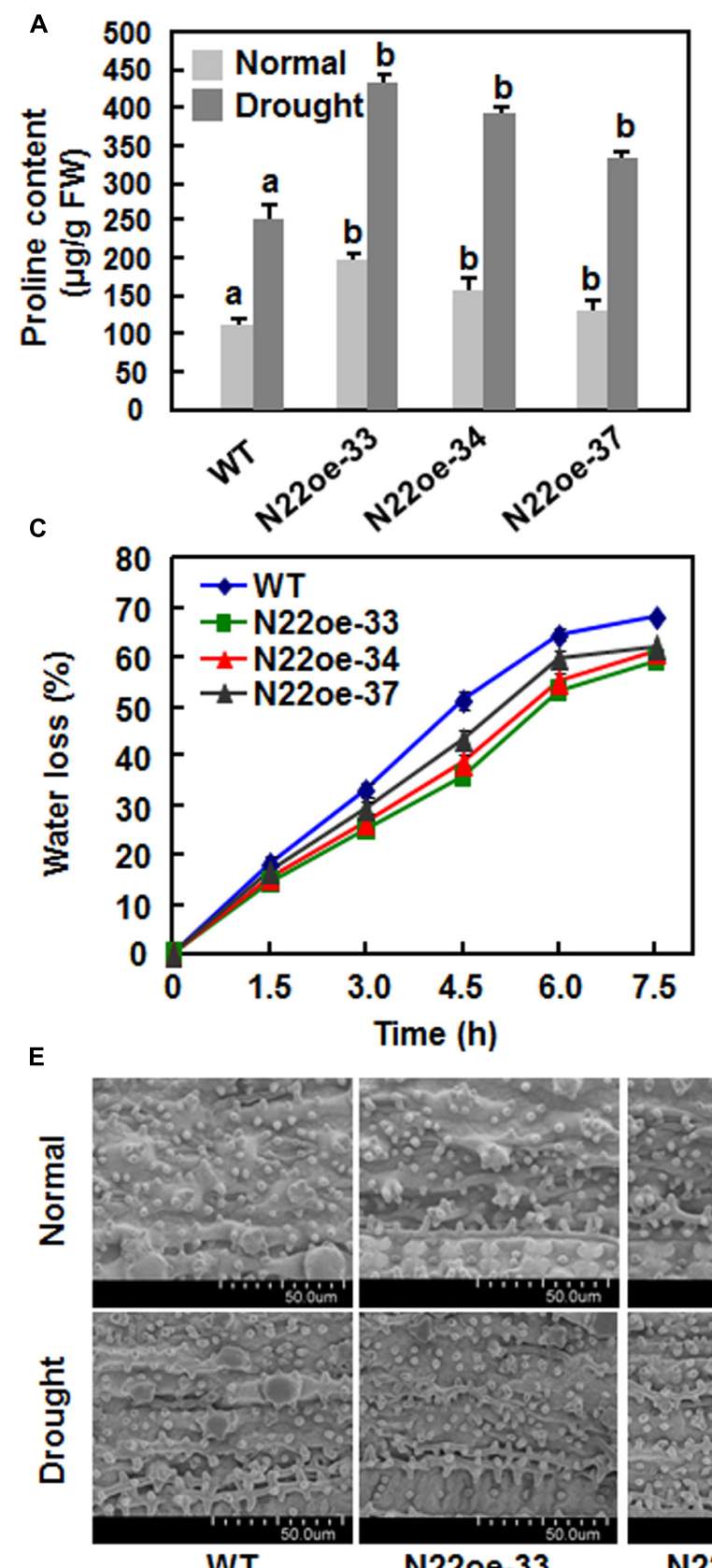

WT

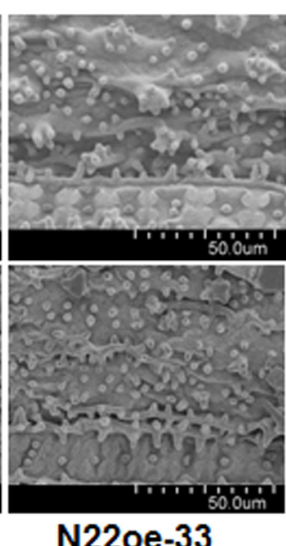

N22oe-33

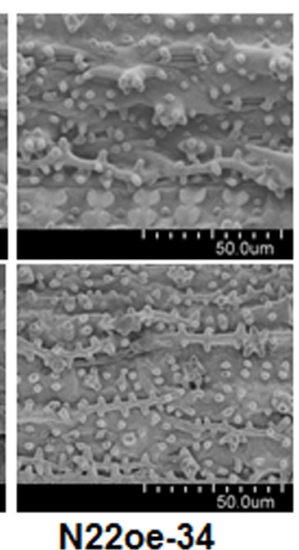

N22oe-34
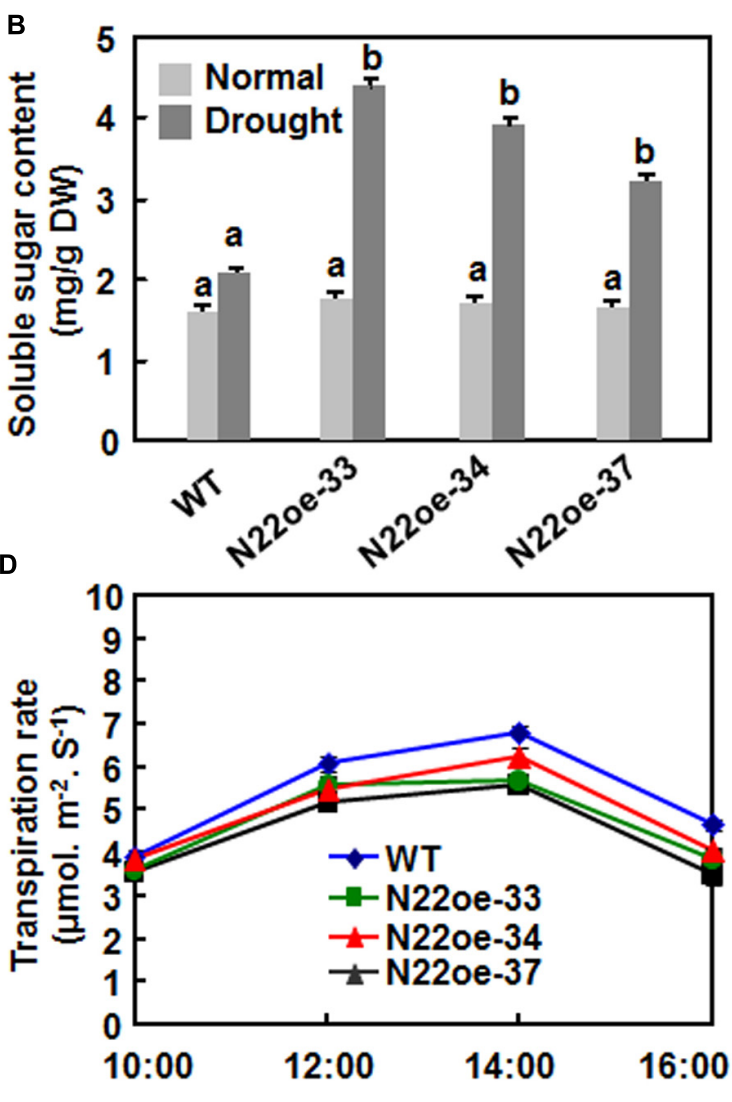

$\mathbf{F}$

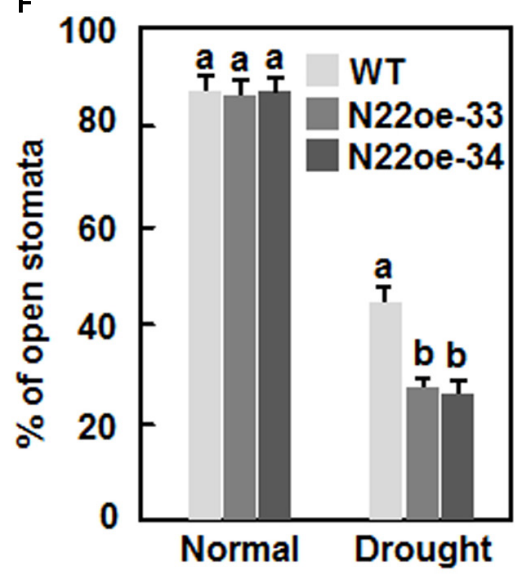

FIGURE 7 | Physiological changes inN22oe plants. (A) Proline contents in leaves of the N22oe and WT plants grown under normal and drought stress condition. (B) Soluble sugar contents in leaves of the N22oe and WT plants grown under normal and drought stress condition. Four-week-old plants were subjected for drought stress treatment by stopping watering and leaf samples were collected at 6 days after drought treatment. (C) Rates of water loss in detached leaves of the N22oe and WT plants. Leaves were detached from 4-week-old N22oe and WT plants grown under normal conditions and placed on lab bench for drought treatment. Leaf samples were collected at indicated time points and subjected for measuring water loss. (D) Transpiration rate of the N220e and WT plants. Transpiration rates in six flag leaves of 3-month-old N22oe and WT plants grown under greenhouse condition were determined by Li-6400 instrument at indicated time points. (E) Stomatal aperture of the N22oe and WT plants grown under normal and drought condition. Scale bar $=50 \mu \mathrm{M}$. (F) Percentage of open stomata in leaves of the N22oe and WT plants grown under normal and drought condition. Data presented in (A-D,F) are the mean \pm SD from three independent experiments and different letters above the columns indicate significant differences at $p \leq 0.05$ level with corresponding WT.

et al., 2006; Redillas et al., 2012), ONAC068 (OsNAC4; Kaneda et al., 2009), ONAC048 (SNAC2/OsNAC6; Nakashima et al., 2007; $\mathrm{Hu}$ et al., 2008), ONAC009 (OsNAC5; Takasaki et al., 2010;
Song et al., 2011; Jeong et al., 2013), ONAC058 (OsNAP; Zhou et al., 2013; Chen et al., 2014; Liang et al., 2014), ONAC122 (OsNAC10; Jeong et al., 2010; Sun et al., 2013), ONAC131 (Sun 


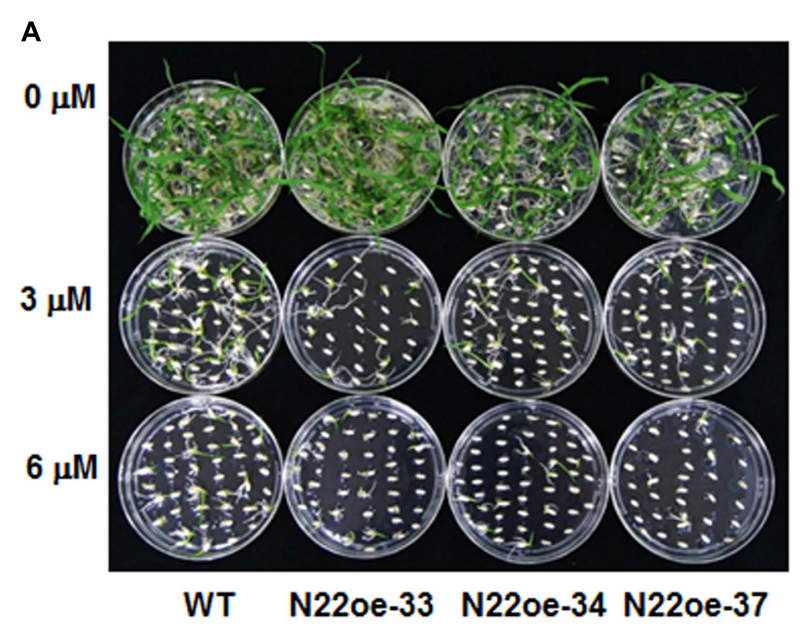

C
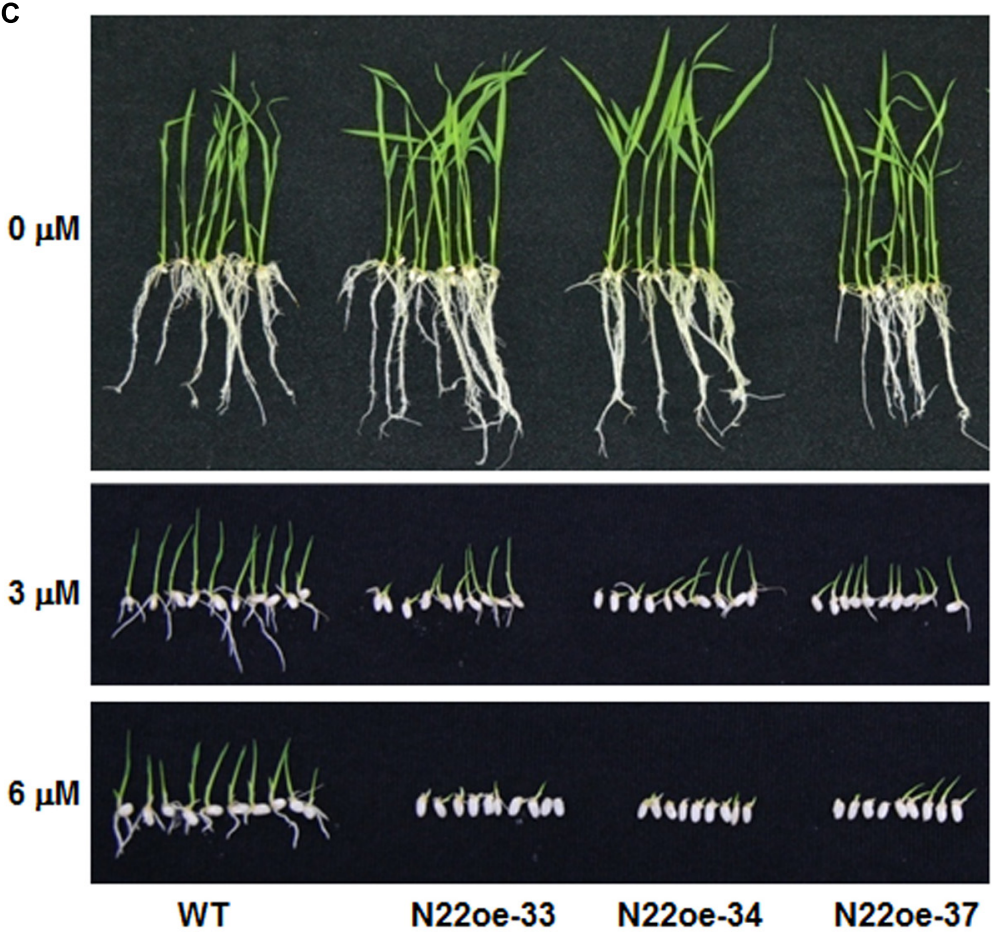
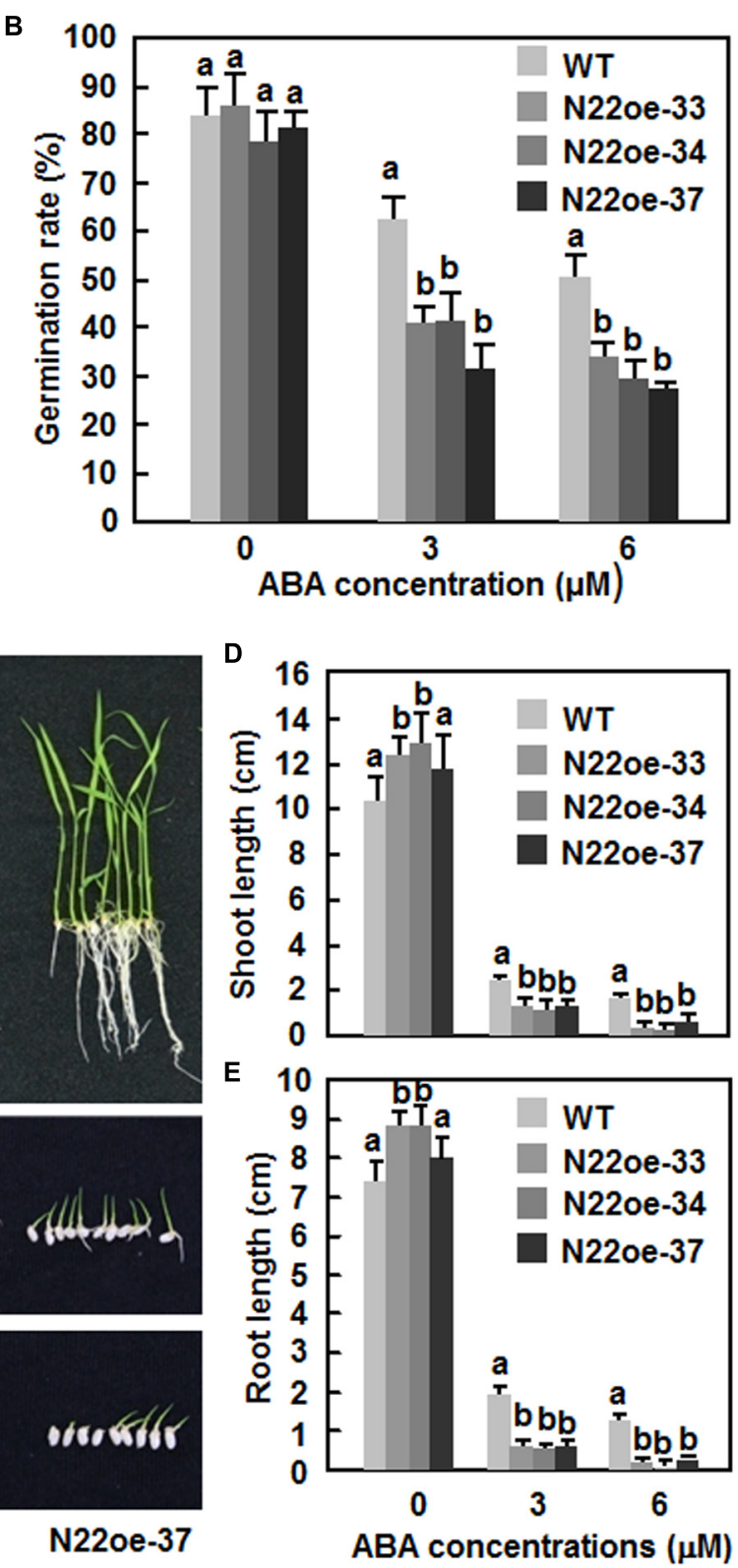

FIGURE 8 | Increased ABA sensitivity of the N22oe seedlings. (A,C) Growth performance and (B) germination rate of the N22oe and WT seedlings on 1/2 MS medium supplemented with or without different concentrations of ABA. Photos were taken at 6 days after germination. (D,E) Shoot and root lengths of the N22oe and WT seedlings grown on 1/2 MS medium supplemented with or without different concentrations of ABA. The shoot and root lengths were measured at 6 days after germination. Data presented in $(\mathbf{D}, \mathbf{E})$ are the mean \pm SD from three independent experiments and different letters above the columns indicate significant differences at $p \leq 0.05$ level with corresponding WT.

et al., 2013), ONAC054 (RIM1; Yoshii et al., 2009), ONAC045 (Zheng et al., 2009), and ONAC017 (OsNAC111; Yokotani et al., 2014) have been shown to play important roles in abiotic and biotic stress responses; however, the biological function of most ONAC genes is not known. In the preset study, we demonstrated that ONAC022 is a stress-responsive NAC with transcriptional activator activity and play an important role in drought and salt stress tolerance in rice. The Arabidopsis ANAC036, the closest ortholog of ONAC022, was found to be involved in the growth of leaf cells (Kato et al., 2010) but its role in abiotic stress response remains unclear.

Our previous comprehensive analysis using publicly available microarray expression data identified a total of 63 ONAC genes, including ONAC022, that exhibited overlapping expression patterns in rice under various abiotic (e.g., salt and drought) and biotic (e.g., infection by fungal, bacterial, and viral pathogens) stresses (Sun et al., 2015). In this study, we further verified that the expression of ONACO22 was induced significantly by 


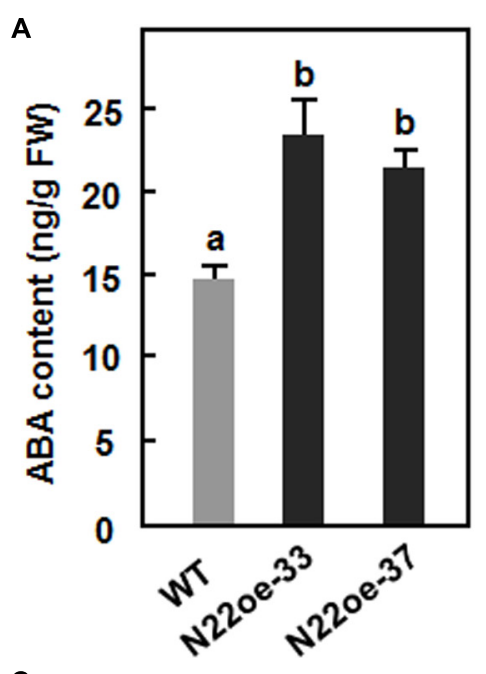

C

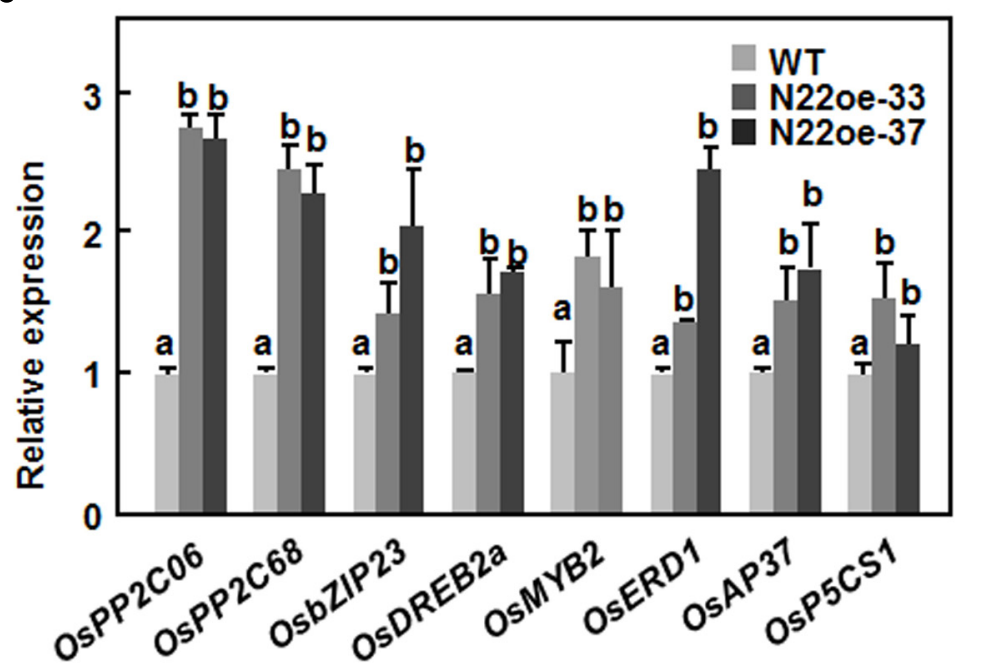

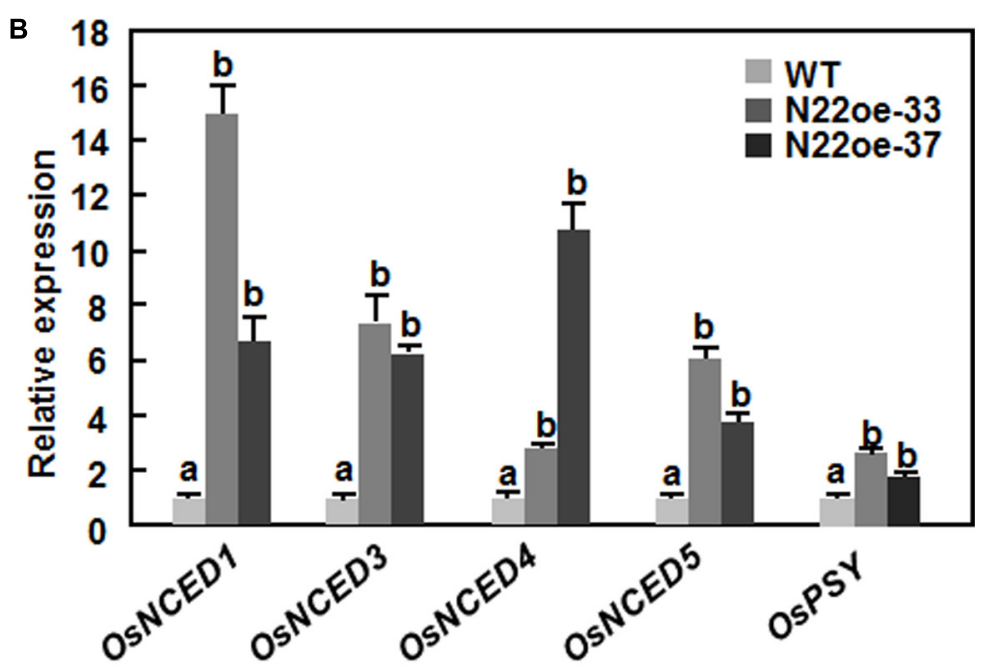

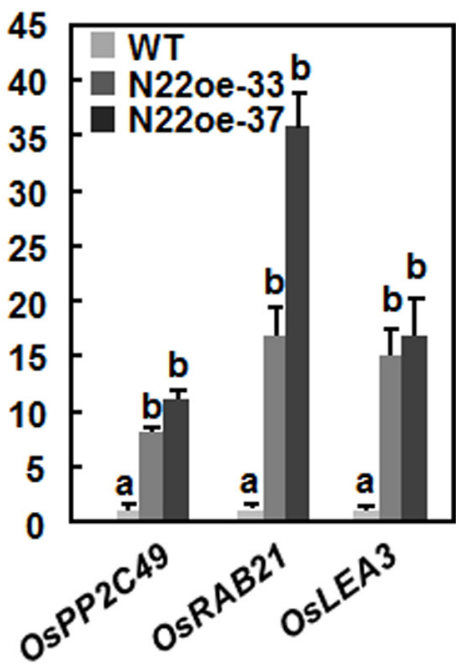

FIGURE 9 | Increased ABA content and upregulated expression of ABA biosynthetic and stress-responsive genes in N22oe plants. (A) Increased ABA contents in the N22oe plants. (B) Expression of ABA biosynthesis-related genes in the N22oe plants. (C) Expression of stress-responsive genes in the N22oe plants. Leaf samples were collected from 30-day-old seedlings grown under normal condition and subjected for analyses of ABA content and gene expression. ABA in leaf samples was extracted and quantified by HPLC. FW, fresh weight. Expression of the ABA biosynthetic and stress-responsive genes was analyzed by qRT-PCR and relative expression levels were shown as folds of the level of the actin gene. Data presented are the mean \pm SD from three independent experiments and different letters above the columns indicate significant differences at $p \leq 0.05$ level with corresponding WT.

drought, salt, and ABA (Figure 2), indicating that ONAC022 can respond to multiple environmental cues. This is partially supported by the fact that the promoter region of the ONAC022 gene contains several stress-related cis-elements such as ABREs and GCC box (Figure 1C), which are present in promoters of stress-responsive genes that are regulated by DREB and ERF TFs, respectively (Yamaguchi-Shinozaki and Shinozaki, 2005, 2006; Mizoi et al., 2012; Licausi et al., 2013). The ONAC022 protein can bind specifically to a canonical NAC recognition cis-element fragment wNACRS in vitro (Xie et al., 2000; Trans et al., 2004) and has transcriptional activity that is dependent on its C-terminal (Figures 3B,C). This is in agreement with a common knowledge that NAC proteins have a C-terminal transcription activation domain (Hu et al., 2006; Takasaki et al., 2010; Liang et al.,
2014). The C-terminal region of ONAC022 contains a newly identified $\mathrm{C} 1$ domain (Figure 1A), which comprises the putative NAC subdomain E and its immediately downstream sequence, indicating that the $\mathrm{C} 1$ domain in ONAC022 may be involved in the DNA-binding ability (Duval et al., 2002; Kato et al., 2010).

The observations that the N22oe plants showed improved drought and salt stress tolerance as revealed by higher survival ratio and better growth performance under drought and salt stress condition (Figures 5 and 6) demonstrate that ONAC022 is a positive regulator of drought and salt stress tolerance in rice. Several other rice NAC genes including ONAC001 (SANC1/OsNAC9; Hu et al., 2006; Redillas et al., 2012), ONAC048 (SNAC2/OsNAC6; Hu et al., 2008; Takasaki et al., 2010; Song et al., 2011; Jeong et al., 2013), ONAC009 (OsNAC6; Nakashima 
et al., 2007), ONAC122 (OsNAC10; Jeong et al., 2010), ONAC058 (OsNAP; Chen et al., 2014), and ONAC045 (Zheng et al., 2009) were previously reported to play positive roles in improving drought and salt tolerance when overexpressed in transgenic rice. The mechanisms responsible for the improved drought and salt tolerance in the N22oe plants can be, at least partially, explained by several morphological, physiological, and biochemical changes observed in the present study. Firstly, accumulation of compatible solutes such as soluble sugars and free proline, which act as osmolytes to facilitate osmo-regulation, molecular chaperones to stabilize proteins or regulators of the antioxidant system, is a common phenomenon in response to abiotic stress (Liu and Zhu, 1997; Garg et al., 2002). It was observed that the N22oe plants accumulated higher levels of free proline under normal condition and high levels of free proline and soluble sugars under drought stress condition, than the WT plants (Figures 7A,B). High levels of free proline in the N22oe plants may due to an upregulated expression of OsP5CS1 (Figure 9C), whose overexpression in transgenic rice led to stress-induced accumulation of free proline and increased abiotic stress tolerance (Zhu et al., 1998). Thus, increased accumulation of free proline and soluble sugars in the N22oe plants may partially account for the improved drought and salt tolerance. In this context, similar observations have also been observed in transgenic rice overexpressing ONAC009 (OsNAC5) and ONAC058 (OsNAP; Song et al., 2011; Chen et al., 2014). Secondly, reduced rates of water loss in detached leaves, decreased transpiration rate and increased stomatal closure in leaves of whole plants were observed in the N22oe plants (Figures 7C,D), which may be the factors that contribute to the improved drought and salt tolerance in the N22oe plants. It is well known that stomatal behavior and/or stomatal density on leaves of plants affect greatly the rates of water loss and transpiration under drought stress condition. For example, it was previously found that increased stomatal closure at the early stage of drought stress was an important factor causing reduced rates of transpiration/water loss and improved drought tolerance in the ONAC002 (SNAC1)-overexpressing rice (Hu et al., 2006). SNAC1 was induced strongly in guard cells of rice plants, suggesting that increased stomatal closure is likely a target of regulation by ONAC002 (SNAC1; Hu et al., 2006). However, whether ONAC022 plays a role in regulating stomatal behavior under drought stress condition and density and thereby contributes to the increased drought tolerance of the N22oe plants need to be examined further. Thirdly, it is well accepted that root system size correlates with the tolerance to water stress and that a longer root system should facilitate water absorption from soils and thus strengthen drought tolerance under water-deficit conditions. For example, drought-resistant rice varieties have a larger and more highly branched root system than drought-sensitive varieties (Price et al., 1997). We also noted that the N22oe plants had larger root system, as revealed by longer roots and more lateral roots than the WT plants, when grown under salt stress condition (Figure 6), indicating a possible role for ONAC022 in regulating root system under stress condition and thereby improving stress tolerance. This is consistent with several observations that overexpression of ONAC122 (OsNAC10; Jeong et al., 2010), ONAC002 (OsNAC9;
Redillas et al., 2012), and ONAC009 (OsNAC5; Jeong et al., 2013) in transgenic rice and ONAC002 (SNAC1; Liu et al., 2014) in transgenic cotton resulted in enhanced root system or altered root architecture involving an enlarged stele and aerenchyma but is contrary to the observation that the ONAC002 (SNAC1)overexpressing rice plants had no difference from WT plants in terms of root depth and volume (Hu et al., 2006).

Abscisic acid plays a critical role in regulating abiotic stress response in plants. In the present study, our results suggest that ONAC022 may function as a positive regulator of stress response through modulating an ABA-mediated pathway to improve drought and salt tolerance in the N22oe rice. This hypothesis is supported by several lines of evidence presented in this study. Firstly, expression of ONACO22 was significantly induced by exogenous ABA (Figure 2C), similar to several previously reported stress-responsive rice NAC genes such as ONAC122 (OsNAC10; Jeong et al., 2010), ONAC048 (SNAC2/OsNAC6; Nakashima et al., 2007; Hu et al., 2008), ONAC009 (OsNAC5; Takasaki et al., 2010; Song et al., 2011), ONAC058 (OsNAP; Chen et al., 2014; Liang et al., 2014), and ONAC002 (SNAC1; Hu et al., 2006). Secondly, the N22oe plants exhibited an increased ABA sensitivity than the WT in terms of seed germination and seedling growth (Figure 8). This is consistent with the observations that overexpression of ONAC058 (OsNAP; Chen et al., 2014), ONAC002 (SNAC1; Hu et al., 2006), and ONAC048 (SNAC2; Hu et al., 2008) in transgenic rice led to improved abiotic stress tolerance and hypersensitivity to exogenous ABA. Thirdly, the endogenous ABA content in the N22oe plants was higher than that in WT plants (Figure 9A), accompanying with upregulated expression of some of the ABA biosynthesis-related genes such as OsNCEDs (Figure 9B), which is generally considered to be the rate-limiting step in the stress-induced ABA biosynthesis pathway (Qin and Zeevaart, 1999; Taylor et al., 2000; Iuchi et al., 2001). These data indicate that overexpression of ONAC022 may influence the biosynthesis of $\mathrm{ABA}$ via modulating directly or indirectly the expression of ABA biosynthesis-related genes. This is similar to the observations that the root-specific overexpression of ONAC002 (OsNAC9) and ONAC122 (OsNAC10) significantly upregulated the expression level of OsNCEDs in transgenic rice (Jeong et al., 2010; Redillas et al., 2012) and that overexpression of OsMYB481, a stress-responsive MYB TF, led to a significant increase in expression of $\mathrm{ABA}$ biosynthetic genes and a high level of the endogenous $A B A$ in transgenic rice plants (Xiong et al., 2014). Generally, as a critical stress hormone, high level of the endogenous ABA might strengthen and/or accelerate stress response and thus correlates with improved abiotic stress tolerance. For example, mutation in OsDSM2/OsBCH1, one of three putative $\beta$-carotene hydroxylases that are predicted for the biosynthesis of ABA precursor zeaxanthin, resulted in reduced level of the endogenous $\mathrm{ABA}$ and decreased drought stress tolerance (Du et al., 2010), whereas RNAi-mediated suppression of OsABA80x3, one of three ABA $8^{\prime}$-hydroxylases involved in catabolism of $\mathrm{ABA}$, led to a high level of the endogenous $\mathrm{ABA}$ and improved drought stress tolerance (Cai et al., 2015). Lastly, the increased endogenous $\mathrm{ABA}$ levels in plants will generally initiate ABA-mediated pathway that regulates the expression of many stress-responsive genes (Xiong et al., 2002). In the present 
study, we observed that a large number of the stress-responsive genes were differentially expressed in N22oe plants (Figure 9C) and the expression of some early ABA signaling and regulatory genes such as OsPP2C06/49/68 (Singh et al., 2010), OsbZIP23 (Xiang et al., 2008), OsDREB2a (Dubouzet et al., 2003), OsMYB2 (Yang et al., 2012), and OsAP37 (Oh et al., 2009) and late ABA-responsive stress-related genes such as OsRAB21 (Xiong et al., 2014), OsLEA3 (Xiao et al., 2007; Duan and Cai, 2012), and OsP5CS1 (Xiong et al., 2014) was significantly upregulated in the N22oe plants (Figure 9C), demonstrating an activated ABA-mediated pathway in the transgenic plants. Overall, these data suggest that ONAC022 in the N22oe plants may accelerate the ABA synthesis via modulating the expression of the ABA biosynthesis-related genes and thus activate an ABA-mediated pathway to regulate the expression of stress-responsive genes. However, it cannot be ruled out the possibility that ONAC022 regulates abiotic stress response through an ABA-independent pathway because the expression of OsERD1, which is known to be involved in the ABAindependent pathway (Shinozaki and Yamaguchi-Shinozaki, 2007), was also upregulated significantly in the N22oe plants (Figure 9C).

Growth retardation has been observed in transgenic rice plants overexpressing stress-responsive TFs such as ONAC048 (OsNAC6) in rice (Nakashima et al., 2007). In the present study, we also noticed that the N22oe plants showed a stunted growth phenotype and smaller panicles compared with the WT plants (Figure 4), indicating that overexpression of ONAC022 in transgenic rice has adverse effects on growth and yield formation. This is similar to the observation that overexpression of ANAC036, the closest ortholog of rice ONAC022, in Arabidopsis led to a dwarf phenotype by reducing cell size in leaves and stems (Kato et al., 2010) but differs from several reports that overexpression of ONAC058 (OsNAP), ONAC002 (OsNAC9), and ONAC122 (OsNAC10) in transgenic rice had no significant effects on growth under normal condition and improved yield production under drought stress condition (Jeong et al., 2010; Redillas et al., 2012; Chen et al., 2014). The fact that a large portion of genes encoding proteins involved in developmental and reproduction processes were differentially expressed in N22oe plants (Supplementary Figure S1) supports the involvement of ONACO22 in rice growth and development. However, the adverse effects of ONAC022 on rice growth and panicle development may be due to re-allocation of energy between stress tolerance and normal growth/development, and the mechanisms need to be further investigated.

\section{REFERENCES}

Ariel, F. D., Manavella, P. A., Dezar, C. A., and Chan, R. L. (2007). The true story of the HD-Zip family. Trends Plant Sci. 12, 419-426.

Bailey, R. W. (1958). Reaction of pentoses with anthrone. Biochem. J. 68, 669-672. doi: $10.1042 /$ bj0680669

Bates, L. S., Waldren, R. P., and Teare, I. D. (1973). Rapid determination of free proline for water-stress studies. Plant Soil 39, 205-207. doi: 10.1016/j.dental.2010.07.006
In summary, results presented in this study demonstrate that ONAC022 functions as a stress-responsive transcriptional activator and overexpression of ONAC022 in transgenic rice can significantly improve drought and salt stress tolerance through an ABA-mediated pathway. Our findings presented in this study, together with several previous reports (Hu et al., 2006, 2008; Jeong et al., 2010; Chen et al., 2014), demonstrate that overexpression of a single regulatory gene such as ONACO22 is a promising strategy to improve the abiotic stress tolerance in rice and other commercially important crops. However, functional analysis with knockout mutants is necessary to determine whether ONAC022 is required for abiotic stress tolerance in rice. Further investigations on the identification of ONACO22regulated target genes will be helpful to elucidate the mechanism of ONAC022 in regulating abiotic stress tolerance. As the N22oe plants gain an improved drought and salt tolerance with a penalty on grain production under unstressed condition, it is necessary to evaluate in detail their performance on abiotic stress tolerance and grain yield under natural or stressed conditions before consideration of their potential use as novel materials in breeding program.

\section{AUTHOR CONTRIBUTIONS}

YH, HZ, DL, and FS designed the research; YH, HZ, LH, and DL performed the experiments; YH and FS wrote the manuscript.

\section{ACKNOWLEDGMENTS}

We are grateful to Dr. Zejian Guo (Department of Plant Pathology, China Agricultural University, China) for providing the pCoU vector, and Dr. Michael Goodin (Department of Plant Pathology, University of Kentucky, USA) for providing the H2BRFP N. benthamiana line. This study was supported by the National High-Tech R \& D Program (No. 2012AA101505), the National Natural Science Foundation (No. 31272028 and No. 30971880), the National Transgenic Major Project of China (No. 2011ZX08009-003-001), and the Research Fund for the Doctoral Program of Higher Education of China (20120101110070).

\section{SUPPLEMENTARY MATERIAL}

The Supplementary Material for this article can be found online at: http://journal.frontiersin.org/article/10.3389/fpls.2016.00004

Cai, S., Jiang, G., Ye, N., Chu, Z., Xu, X., Zhang, J., et al. (2015). A key ABA catabolic gene, OsABA8ox3, is involved in drought stress resistance in rice. PLoS ONE 10:e0116646. doi: 10.1371/journal.pone.0116646

Castilhos, G., Lazzarotto, F., Spagnolo-Fonini, L., Bodanese-Zanettini, M. H., and Margis-Pinheiro, M. (2014). Possible roles of basic helix-loop-helix transcription factors in adaptation to drought. Plant Sci. 223, 1-7. doi: 10.1016/j.plantsci.2014.02.010

Chakrabarty, R., Banerjee, R., Chung, S. M., Farman, M., Citovsky, V., Hogenhout, S. A., et al. (2007). pPSITE vectors for stable integration or transient 
expression of autofluorescent protein fusions in plants: probing Nicotiana benthamiana-virus interactions. Mol. Plant Microbe Interact. 20, 740-750. doi: 10.1094/MPMI-20-7-0740

Chen, L., Song, Y., Li, S., Zhang, L., Zou, C., and Yu, D. (2012). The role of WRKY transcription factors in plant abiotic stresses. Biochim. Biophys. Acta 1819, 120-128. doi: 10.1016/j.bbagrm.2011.09.002

Chen, X., Wang, Y. F., Lv, B., Li, J., Luo, L. Q., Lu, S. C., et al. (2014). The NAC family transcription factor OsNAP confers abiotic stress response through the ABA pathway. Plant Cell Physiol. 55, 604-619. doi: 10.1093/pcp/pct204

Du, H., Wang, N. L., Cui, F., Li, X. H., Xiao, J. H., and Xiong, L. Z. (2010). Characterization of the $\beta$-Carotene hydroxylase gene DSM 2 conferring drought and oxidative stress resistance by increasing xanthophylls and abscisic acid synthesis in rice. Plant Physiol. 154, 1304-1318. doi: 10.1104/pp.110.163741

Duan, J. L., and Cai, W. M. (2012). OsLEA3-2, an abiotic stress induced gene of rice plays a key role in salt and drought tolerance. PLOS ONE 7:e45117. doi: 10.1371/journal.pone.0045117

Dubouzet, J. G., Sakuma, Y., Ito, Y., Kasuga, M., Dubouzet, E. G., Miura, S., et al. (2003). OsDREB genes in rice, Oryza sativa L., encode transcription activators that function in drought, high salt and cold-responsive gene expression. Plant J. 33, 751-763. doi: 10.1046/j.1365-313X.2003.01661.x

Duval, M., Hsieh, T. F., Kim, S. Y., and Thomas, T. L. (2002). Molecular characterization of AtNAM: a member of Arabidopsis NAC domain superfamily. Plant Mol. Biol. 50, 237-248. doi: 10.1023/A:1016028530943

Fang, Y. J., You, J., Xie, K., Xie, W. B., and Xiong, L. Z. (2008). Systematic sequence analysis and identification of tissue-specific or stress-responsive genes of NAC transcription factor family in rice. Mol. Genet. Genomics 280, 547-563. doi: 10.1007/s00438-008-0386-6

Fu, J. H., Chu, J. F., Sun, X. H., Wang, J. D., and Yan, C. Y. (2012). Simple, rapid and simultaneous assay of multiple carboxyl containing phytohormones in wounded tomatoes by UPLC-MS/MS using single SPE purification and isotope dilution. Anal. Sci. 28, 1081-1087. doi: 10.2116/analsci.28.1081

Garg, A. K., Kim, J. K., Owens, T. G., Ranwala, A. P., Choi, Y. D., Kochian, L. V., et al. (2002). Trehalose accumulation in rice plants confers high tolerance levels to different abiotic stresses. Proc. Natl. Acad. Sci. U.S.A. 99, 15898-15903. doi: 10.1073/pnas.252637799

Golldack, D., Li, C., Mohan, H., and Probst, N. (2014). Tolerance to drought and salt stress in plants: unraveling the signaling networks. Front. Plant Sci. 5:151. doi: 10.3389/fpls.2014.00151

Hu, H. H., Dai, M. Q., Yao, J. L., Xiao, B. Z., Li, X. H., Zhang, Q. F., et al. (2006). Overexpressing a NAM, ATAF, and CUC (NAC) transcription factor enhances drought resistance and salt tolerance in rice. Proc. Natl. Acad. Sci. U.S.A. 103, 12987-12992. doi: 10.1073/pnas.0604882103

$\mathrm{Hu}, \mathrm{H}$. , and Xiong, L. (2014). Genetic engineering and breeding of droughtresistant crops. Annu. Rev. Plant Biol. 65, 715-741. doi: 10.1146/annurevarplant-050213-040000

Hu, H. H., You, J., Fang, Y. J., Zhu, X. Y., Qi, Z. Y., and Xiong, L. Z. (2008). Characterization of transcription factor gene SNAC2 conferring cold and salt tolerance in rice. Plant Mol. Biol. 67, 169-181. doi: 10.1007/s11103-008-9309-5

Iuchi, S., Kobayashi, M., Taji, T., Naramoto, M., Seki, M., Kato, T., et al. (2001). Regulation of drought tolerance by gene manipulation of 9-cis-epoxycarotenoid dioxygenase, a key enzyme in abscisic acid biosynthesis in Arabidopsis. Plant J. 27, 325-333. doi: 10.1046/j.1365-313x.2001.01096.x

Jabeen, Z., Hussain, N., Han, Y., Shah, M. S., Zeng, F. R., Zeng, J. B., et al. (2014). The differences in physiological responses, ultrastructure changes, and $\mathrm{Na}+$ subcellular distribution under salt stress among the barley genotypes differing in salt tolerance. Acta Physiol. Plant. 36, 2397-2407. doi: 10.1007/s11738-0141613-x

Jeong, J. S., Kim, Y. S., Baek, K. H., Jung, H., Ha, S. H., Choi, Y. D., et al. (2010). Root-specific expression of OsNAC10 improves drought tolerance and grain yield in rice under field drought conditions. Plant Physiol. 153, 185-197. doi: 10.1104/pp.110.154773

Jeong, J. S., Kim, Y. S., Redillas, M. F. R., Jang, G., Jung, H., Bang, S. W., et al. (2013). OsNAC5 overexpression enlarges root diameter in rice plants leading to enhanced drought tolerance and increased grain yield in the field. Plant Biotechnol. J. 11, 101-114. doi: 10.1111/pbi.12011

Jiang, Y., and Deyholos, M. K. (2006). Comprehensive transcriptional profiling of $\mathrm{NaCl}$-stressed Arabidopsis roots reveals novel classes of responsive genes. BMC Plant Biol. 6:25. doi: 10.1186/1471-2229-6-25
Kaneda, T., Taga, Y., Takai, R., Iwano, M., Matsui, H., Takayama, S., et al. (2009). The transcription factor OsNAC4 is a key positive regulator of plant hypersensitive cell death. EMBO J. 28, 926-936. doi: 10.1038/emboj.2009.39

Kato, H., Motomura, T., Komeda, Y., Saito, T., and Kato, A. (2010). Overexpression of the NAC transcription factor family gene ANAC036 results in a dwarf phenotype in Arabidopsis thaliana. J. Plant Physiol. 167, 571-577. doi: 10.1016/j.jplph.2009.11.004

Kiyosue, T., Yamaguchi-Shinozaki, K., and Shinozaki, K. (1994). Cloning of cDNAs for genes that are early-responsive to dehydration stress (ERDs) in Arabidopsis thaliana L: identification of three ERDs as HSP cognate genes. Plant Mol. Biol. 25, 791-798. doi: 10.1007/BF00028874

Le, D. T., Nisjiyama, R., Watanabe, Y., Mochida, K., Yamaquchi-Shinozaki, K., Shinozaki, K., et al. (2011). Genome-wide survey and expression analysis of the plant-specific NAC transcription factor family in soybean during development and dehydration stress. DNA Res. 8, 263-276. doi: 10.1093/dnares/d sr015

Liang, C. Z., Wang, Y. Q., Zhu, Y., Tang, J. Y., Hu, B., Liu, L. C., et al. (2014). OsNAP connects abscisic acid and leaf senescence by fine-tuning abscisic acid biosynthesis and directly targeting senescence-associated genes in rice. Proc. Natl. Acad. Sci. U.S.A. 111, 10013-10018. doi: 10.1073/pnas.13215 68111

Licausi, F., Ohme-Takagi, M., and Perata, P. (2013). APETALA2/Ethylene Responsive Factor (AP2/ERF) transcription factors: mediators of stress responses and developmental programs. New Phytol. 199, 639-649. doi: $10.1111 /$ nph.12291

Liu, G. Z., Li, X. L., Jin, S. X., Liu, X. Y., Zhu, L. F., Nie, Y. C., et al. (2014). Overexpression of rice NAC gene SNAC1 improves drought and salt tolerance by enhancing root development and reducing transpiration rate in transgenic cotton. PLoS ONE 9:e86895. doi: 10.1371/journal.pone.0086895

Liu, J., and Zhu, J. K. (1997). Proline accumulation and salt-stress-induced gene expression in a salt-hypersensitive mutant of Arabidopsis. Plant Physiol. 114, 591-596. doi: 10.1104/pp.114.2.591

Mizoi, J., Shinozaki, K., and Yamaguchi-Shinozaki, K. (2012). AP2/ERF family transcription factors in plant abiotic stress responses. Biochim. Biophys. Acta 1819, 86-96. doi: 10.1016/j.bbagrm.2011.08.004

Nakashima, K., Tran, L. P., Nguyen, D. V., Fujita, M., Maruyama, K., Todaka, D., et al. (2007). Functional analysis of a NAC-type transcription factor OsNAC6 involved in abiotic and biotic stress-responsive gene expression in rice. Plant J. 51, 617-630. doi: 10.1111/j.1365-313X.2007.03168.x

Nakashima, K., Yamaguchi-Shinozaki, K., and Shinozaki, K. (2014). The transcriptional regulatory network in the drought response and its crosstalk in abiotic stress responses including drought, cold, and heat. Front. Plant Sci. 5:170. doi: 10.3389/fpls.2014.00170

Nuruzzaman, M., Manimekalai, R., Sharoni, A. M., Satoh, K., Kondoh, H., Ooka, H., et al. (2010). Genome-wide analysis of NAC transcription factor family in rice. Gene 465, 30-44. doi: 10.1016/j.gene.2010.06.008

Nuruzzaman, M., Sharoni, A. M., Satoh, K., Karim, M. R., Harikrishna, J. A., Shimizu, T., et al. (2015). NAC transcription factor family genes are differentially expressed in rice during infections with Rice dwarf virus, Rice black-streaked dwarf virus, Rice grassy stunt virus, Rice ragged stunt virus and Rice transitory yellowing virus. Front. Plant Sci. 6:676. doi: $10.3389 /$ fpls.2015.00676

Nuruzzaman, M., Sharoni, M., and Kikuchi, S. (2013). Roles of NAC transcription factors in the regulation of biotic and abiotic stress responses in plants. Front. Microbiol. 4:248. doi: 10.3389/fmicb.2013.00248

Oh, S. J., Kim, Y. S., Kwon, C. W., Park, H. K., Jeong, J. S., and Kim, J. K. (2009). Overexpression of the transcription factor AP37 in rice improves grain yield under drought conditions. Plant Physiol. 150, 1368-1379. doi: 10.1104/pp.109.137554

Olsen, A. N., Ernst, H. A., Leggio, L. L., and Skriver, K. (2005). NAC transcription factors: structurally distinct, functionally diverse. Trends Plant Sci. 10, 79-87. doi: 10.1016/j.tplants.2004.12.010

Ooka, H., Satoh, K., Doi, K., Nagata, T., Otomo, Y., Murakami, K., et al. (2003). Comprehensive analysis of NAC family genes in Oryza sativa and Arabidopsis thaliana. DNA Res. 10, 239-247. doi: 10.1093/dnares/10.6.239

Price, A. H., Tomos, A. D., and Virk, D. S. (1997). Genetic dissection of root growth in rice (Oryza sativa L.) I: a hydrophonic screen. Theor. Appl. Genet. 95, 132-142. doi: 10.1007/s001220050542 
Puranik, S., Sahu, P. P., Srivastava, P. S., and Prasad, M. (2012). NAC proteins: regulation and role in stress tolerance. Trends Plant Sci. 17, 369-381. doi: 10.1016/j.tplants.2012.02.004

Qin, X., and Zeevaart, J. A. (1999). The 9-cis-epoxycarotenoid cleavage reaction is the key regulatory step of abscisic acid biosynthesis in water-stressed bean. Proc. Natl. Acad. Sci. U.S.A. 96, 15354-15361. doi: 10.1073/pnas.96.26.15354

Redillas, M. C., Jeong, J. S., Kim, Y. S., Jung, H., Wang, S. W., Choi, Y. D., et al. (2012). The overexpression of OsNAC9 alters the root architecture of rice plants enhancing drought resistance and grain yield under field conditions. Plant Biotechnol. J. 10, 792-805. doi: 10.1111/j.1467-7652.2012.00697.x

Rushton, D. L., Tripathi, P., Rabara, R. C., Lin, J., Ringler, P., Boken, A. K., et al. (2012). WRKY transcription factors: key components in abscisic acid signalling. Plant Biotechnol. J. 10, 2-11. doi: 10.1111/j.1467-7652.2011.00634.x

Schonfeld, M. A., Johnson, R. C., and Carver, B. F. (1988). Water relations in winter wheat as drought resistance indicator. Crop Sci. 28, 526-531. doi: 10.2135/cropsci1988.0011183X002800030021x

Shao, H., Wang, H., and Tang, X. (2015). NAC transcription factors in plant multiple abiotic stress responses: progress and prospects. Front. Plant Sci. 6:902. doi: 10.3389/fpls.2015.00902

Shinozaki, K., and Yamaguchi-Shinozaki, K. (2007). Gene networks involved in drought stress response and tolerance. J. Exp. Bot. 58, 221-227. doi: 10.1093/jxb/erl164

Singh, A., Giri, J., Kapoor, S., Tyagi, A. K., and Pandey, G. K. (2010). Protein phosphatase complement in rice: genome-wide identification and transcriptional analysis under abiotic stress conditions and reproductive development. BMC Genomics 11:435. doi: 10.1186/1471-2164-11-435

Song, S. Y., Chen, Y., Chen, J., Dai, X. Y., and Zhang, W. H. (2011). Physiological mechanisms underlying OsNAC5-dependent tolerance of rice plants to abiotic stress. Planta 234, 331-345. doi: 10.1007/s00425-011-1403-2

Sun, L., Huang, L., Hong, Y., Zhang, H., Song, F., and Li, D. (2015). Comprehensive analysis suggests overlapping expression of rice ONAC transcription factors in abiotic and biotic stress responses. Int. J. Mol. Sci. 16, 4306-4326. doi: $10.3390 /$ ijms 16024306

Sun, L. J., Zhang, H., Li, D., Huang, L., Hong, Y., Ding, X. S., et al. (2013). Functions of rice NAC transcriptional factors, ONAC122 and ONAC131, in defense responses against Magnaporthe grisea. Plant Mol. Biol. 81, 41-56. doi: 10.1007/s11103-012-9981-3

Takasaki, H., Maruyama, K., Kidokoro, S., Ito, Y., Fujita, Y., Shinozaki, K., et al. (2010). The abiotic stress-responsive NAC-type transcription factor OsNAC5 regulates stress-inducible genes and stress tolerance in rice. Mol. Genet. Genomics 284, 173-183. doi: 10.1007/s00438-010-0557-0

Taylor, I. B., Burbidge, A., and Thompson, A. J. (2000). Control of abscisic acid synthesis. J. Exp. Bot. 51, 1563-1574. doi: 10.1093/jexbot/51.350.1563

Trans, L. P., Nakashima, K., Sakuma, Y., Simpson, S. D., Fujita, Y., Maruyama, K., et al. (2004). Isolation and functional analysis of Arabidopsis stress-inducible NAC transcription factor that bind to a drought-responsive cis-element in the early responsive to dehydration stress 1 promoter. Plant Cell 16, 2481-2498. doi: $10.1105 /$ tpc. 104.022699

Xiang, Y., Tang, N., Du, H., Ye, H. Y., and Xiong, L. Z. (2008). Characterization of OsbZIP23 as a key player of the basic leucine zipper transcripti0n factor family for conferring abscisic acid sensitivity and salinity and drought tolerance in rice. Plant Physiol. 148, 1938-1952. doi: 10.1104/pp.108.128199

Xiao, B. Z., Huang, Y. M., Tang, N., and Xiong, L. Z. (2007). Over-expression of a LEA gene in rice improves drought resistance under the field conditions. Theor. Appl. Genet. 115, 35-46. doi: 10.1007/s00122-007-0538-9

Xie, Q., Frugis, G., Colgan, D., and Chua, N. H. (2000). Arabidopsis NAC1 transduces auxin signal downstream of TIR1 to promote lateral root development. Genes Dev. 14, 3024-3036. doi: 10.1101/gad.852200
Xiong, H. Y., Li, J. J., Liu, P. L., Duan, J. Z., Zhao, Y., Guo, X., et al. (2014). Overexpression of OsMYB48-1, a novel MYB-Related transcription factor, enhances drought and salinity tolerance in rice. PLOS ONE 9:e92913. doi: 10.1371/journal.pone.0092913

Xiong, L., Schumaker, K. S., and Zhu, J. K. (2002). Cell signaling during cold, drought, and salt stress. Plant Cell 14, S165-S183.

Yamaguchi-Shinozaki, K., and Shinozaki, K. (2005). Organization of cis-acting regulatory elements in osmotic and cold-stress-responsive promoters. Trends Plant Sci. 10, 88-94. doi: 10.1016/j.tplants.2004.12.012

Yamaguchi-Shinozaki, K., and Shinozaki, K. (2006). Transcriptional regulatory networks in cellular response and tolerance to dehydration and cold stresses. Annu. Rev. Plant Biol. 57, 781-803. doi: 10.1146/annurev.arplant.57.032905.105444

Yang, A., Dai, X. Y., and Zhang, W. H. (2012). A R2R3 MYB gene, OsMYB2, is involved in salt, cold and dehydration tolerance in rice. J. Exp. Bot. 63, 2541-2556. doi: 10.1093/jxb/err431

Yokotani, N., Tsuchida-Mayama, T., Ichikawa, H., Mitsuda, N., Ohme-Takagi, M., Kaku, H., et al. (2014). OsNAC111, a blast disease-response transcription factor in rice, positively regulates the expression of defense-related genes. Mol. Plant Microbe Interact. 27, 1027-1034. doi: 10.1094/MPMI-03-140065-R

Yoshii, M., Shimizu, T., Yamazaki, M., Higashi, T., Miyao, A., Hirochika, H., et al. (2009). Disruption of novel gene for a NAC-domain protein in rice confers resistance to Rice dwarf virus. Plant J. 57, 615-625. doi: 10.1111/j.1365313X.2008.03712.x

You, J., Zong, W., Li, X. K., Ning, J., Hu, H. H., Li, X. H., et al. (2013). The SNAC1-targeted gene OsSRO1c modulates stomatal closure and oxidative stress tolerance by regulating hydrogen peroxide in rice. J. Exp. Bot. 64, 569-583. doi: $10.1093 /$ jxb/ers349

Zhang, J., Peng, Y. L., and Guo, Z. J. (2008). Constitutive expression of pathogeninducible OsWRKY31 enhances disease resistance and affects root growth and auxin response in transgenic rice plants. Cell Res. 18, 508-521. doi: 10.1038/cr.2007.104

Zheng, X. N., Zhen, B., Lu, G. J., and Han, B. (2009). Overexpression of a NAC transcription factor enhances rice drought and salt tolerance. Biochem. Biophys. Res. Commun. 379, 985-989. doi: 10.1016/j.bbrc.2008. 12.163

Zhou, Y., Huang, W. F., Liu, L., Chen, T. Y., Zhou, F., and Lin, Y. J. (2013). Identification and functional characterization of a rice NAC gene involved in the regulation of leaf senescence. BMC Plant Biol. 13:132. doi: 10.1186/14712229-13-132

Zhu, B. C., Su, J., Chan, M. C., Verma, D. P. S., Fan, Y. L., and Wu, R. (1998). Over-expression of a $\Delta$-pyrroline- 5 carboxylate synthetase gene and analysis of tolerance to water-stress and salt-stress in transgenic rice. Plant Sci. 139, 41-48. doi: 10.1016/S0168-9452(98)00175-7

Zhu, J. K. (2002). Salt and drought stress signal transduction in plants. Annu. Rev. Plant Biol. 53, 247-273. doi: 10.1146/annurev.arplant.53.091401.143329

Conflict of Interest Statement: The authors declare that the research was conducted in the absence of any commercial or financial relationships that could be construed as a potential conflict of interest.

Copyright (c) 2016 Hong, Zhang, Huang, Li and Song. This is an open-access article distributed under the terms of the Creative Commons Attribution License (CC BY). The use, distribution or reproduction in other forums is permitted, provided the original author(s) or licensor are credited and that the original publication in this journal is cited, in accordance with accepted academic practice. No use, distribution or reproduction is permitted which does not comply with these terms. 